\title{
Adaptive Eulerian framework for boiling and evaporation
}

\author{
Mehdi Khalloufi*, Rudy Valette, Elie Hachem \\ ${ }^{a}$ MINES ParisTech, PSL - Research University, CEMEF - Centre for material forming, \\ CNRS UMR 7635, CS 10207 rue Claude Daunesse, 06904 Sophia-Antipolis Cedex, France.
}

\begin{abstract}
We propose in this work an adaptive Eulerian framework for the simulation of both boiling and evaporation phenomena occurring at the interface of a heated 3D solid immersed in a liquid tank. It simultaneously takes into account the gas-liquid phase changes, the vapor formation and their dynamics, and consequently the 3D quenching or cooling of a heated solid. It uses a Level Set method to separate and to track each phase. The phase change is performed using the balance of heat fluxes at the interface without the use of conforming mesh. Instead, the use of an a posteriori error estimate leading to highly stretched anisotropic elements at the interface enables to drastically reduce errors on computed jumps. This avoids the need of interface reconstruction or interpolation procedure. Finally, a Variational Multiscale solver for the Navier-Stokes equations is extended with implicit treatment of the surface tension. A series of $2 \mathrm{D}$ and $3 \mathrm{D}$ problems are solved to highlight the efficiency and the accuracy of the proposed framework. The cooling of an immersed solid is also presented and shows good agreement with experimental data. To the best of our knowledge, direct numerical simulations of quenching using an Eulerian framework with boiling and evaporation have never been considered.
\end{abstract}

Keywords: Phase change, Multiphase flows, Immersed method, Level Set, Anisotropic mesh adaptation, Stefan problem

\section{1. INTRODUCTION}

An accurate and robust simulation of boiling phenomena is still an ongoing challenge. Indeed, the complexity of boiling lies in the wide range of scales to consider and in the physics involved. From the nucleation of vapor bubbles to film boiling and bath hydrodynamics, one needs to consider scales from $\mu m$ to $m$. Indeed, in industrial processes, such as quenching of a solid hot metallic part, the variety of configurations and the complexity of the surrounding flow must also be taken into account as they have direct impacts on gradients of mechanical properties, microstructure and residual stresses.

\footnotetext{
* Corresponding author

Email addresses: mehdi.khalloufi@mines-paristech.fr, khalloufi.mehdi@gmail.com (Mehdi Khalloufi ), rudy.valette@mines-paristech.fr (Rudy Valette), elie.hachem@mines-paristech.fr (Elie Hachem)
}

Preprint submitted to Journal of Computational Physics

September 6, 2019 
The classical well known methods for thermal treatment during the cooling of a solid generally refer to the use of experimentally deduced heat transfer coefficients. However, these latter are only useful for a particular configuration in term of geometry, orientation of the surface, range of temperature and flow motion. Therefore, heat transfer coefficients are not suitable to analyze different industrial processes and cannot be generalized to all configurations. Only a direct numerical simulation can take into account all the physics and all the scales involved in boiling.

An abundant research has been devoted to the modeling of the boiling and the evaporation phenomena. The approaches to simulate these phenomena can be summarized into two categories that depend on the thickness of the interface: sharp interface and diffuse interface. The sharp interface approach considers the presence of different phases that interact through the interface using boundary conditions. Within this approach, two methods are commonly used. First, in interface tracking methods, the interface is represented by a set of nodes. This set of nodes moves accordingly to the interface. This requires particular mesh movements at each iteration and also requires a special treatment of complex topological configurations [1-5]. Alternatively, interface capturing methods consist in a implicit definition of the interface. A volume fraction (Volume-Of-Fluid) or a signed distance function (Level Set) is convected using a transport equation [6-18]. These methods are popular due to the simplicity of implementation and the fact that complex topology changes such as the coalescence of bubbles are handled naturally. A literature review of these methods applied to boiling is given by Kharangate and Mudawar in [19]. Other approaches in the literature can be mentioned. Phase-field approaches seem promising [20-22]. An order parameter is used to distinguish different phases and its evolution gives access to the interface location. The interface is assumed to have a thickness of a few nanometers. However, an equation of state is required for the fluid which introduces complexity from a mathematical and numerical point of view. Furthermore, this approach introduces high-order differential terms that may be stiff and require innovative discretization techniques. We can also mention the fluid mixtures approaches $[23,24]$ considering two compressible phases, where a complete hyperbolic system is derived using conservation equations for each phase. An equation of state that reproduces the phase diagram is usually required to close the system.

We propose in this work an adaptive Eulerian framework for the simulation of both boiling and evaporation phenomena occurring at the interface of a heated 3D solid immersed in a liquid tank. It was shown that an adaptive Eulerian framework is very efficient for the simulation multiphase flows [25-28], compressible and incompressible flows [29] and yield stress fluids [30]. This framework takes into account the gas-liquid phase changes, the vapor formation and their dynamics, and enables consequently the simulation of 3D quenching or cooling of a heated solid. A level set method is used to separate and track the different phases. The phase change is performed using the balance of heat fluxes at the interface without the use of conforming mesh. Instead, the use of an a posteriori error estimate [31-33] leading to highly stretched anisotropic elements at the interface enables to drastically reduce errors on computed jumps. This avoids the need 
of interface reconstruction or interpolation procedure. We derive the Navier-Stokes equations taking into account the mass and energy transfer between phases at the interface. A series of $2 \mathrm{D}$ and $3 \mathrm{D}$ problems are solved to highlight the efficiency and the accuracy of the proposed framework. The cooling of an immersed solid is also presented and shows good agreement with experimental data. To the best of our knowledge, direct numerical simulations of quenching using an Eulerian framework with boiling and evaporation have never been considered.

The paper is structured as follows. In Section 2, we present the governing equations for the fluid motion and the level set transport equation. In Section 3, we recall the main steps of the anisotropic mesh adaptation procedure governed by the length distribution tensor. In Section 4, we provide some numerical results and examples to assess the capability of the proposed method.

\section{Eulerian framework}

This section is devoted to the presentation of the Eulerian framework. First, the interface capturing method is presented. It enables to follow the evolution of the interface between phases and to distribute in space the respective physical properties by defining a mixing law. From this mixing law, a phase change model will be derived and the Navier-Stokes equations will be consequently modified to take into account the phase change model.

\subsection{Level Set approach}

In this section, we describe the level set method used to locate the interface between the liquid phase and the vapor phase. It is a signed distance function and it is a widely used tool in different fields such as crystal growth, image restoration or surface reconstruction [34]. Let $\Omega$ be the whole domain, $\Omega_{l}$ the liquid domain and $\Omega_{v}$ the vapor domain. The level set function is a signed distance function from the interface $\Gamma=\Omega_{l} \cap \Omega_{v}$ defined at each node $\mathrm{X}$ of $\Omega$ as follows:

$$
\alpha(X)= \begin{cases}-\operatorname{dist}(X, \Gamma) & \text { if } X \in \Omega_{l}, \\ 0 & \text { if } X \in \Gamma, \\ \operatorname{dist}(X, \Gamma) & \text { if } X \in \Omega_{v} .\end{cases}
$$

The evolution of the level set function is described by the following transport equation [35]

$$
\frac{\partial \alpha}{\partial t}+u \cdot \nabla \alpha=0
$$

where $u$ is a velocity. The level set, as a distance function, verifies $\|\nabla \alpha\|=1$. However, when the interface is convected by $u$, the level set can lose this property, which may cause numerical instabilities, and needs to be reinitialized to recover it. A common way to reinitialize it, is to solve the following Hamilton-Jacobi equation [35] 


$$
\frac{\partial \alpha}{\partial \tau}+\mathrm{s}(\alpha)(\|\nabla \alpha\|-1)=0,
$$
where $H$ is a smoothed Heaviside function used to obtain a better continuity at the interface and given by

$$
H(\alpha)= \begin{cases}1 & \text { if } \alpha>\varepsilon, \\ \frac{1}{2}\left(1+\frac{\alpha}{\varepsilon}+\frac{1}{\pi} \sin \left(\frac{\pi \alpha}{\varepsilon}\right)\right) & \text { if }|\alpha| \leq \varepsilon, \\ 0 & \text { if } \alpha<-\varepsilon .\end{cases}
$$

75 Here $\varepsilon$ is a small parameter such that $\varepsilon=O\left(h_{\mathrm{im}}\right)$, known as the interface thickness, and $h_{\mathrm{im}}$ is the mesh 76 size in the normal direction to the interface. In the vicinity of the interface, it can be computed using

$$
h_{\mathrm{im}}=\max _{j, l \in K} \nabla \alpha \cdot \mathbf{x}^{j l},
$$

77 where $K$ is the mesh element under consideration and $\mathbf{x}^{j l}=\mathbf{x}^{l}-\mathbf{x}^{j}$ represents the edge connecting the 78 nodes $\mathbf{x}^{j}$ and $\mathbf{x}^{l}$ of $K$.

\subsection{Phase change model}

In this section, we derive a pseudo-compressible model accounting for mass transfer at the interface.

81 From the mass conservation equation, we will quantify the exchange of mass between the vapor and the

$$
\begin{aligned}
\rho\left(\partial_{t} u+u \cdot \nabla u\right)-\nabla \cdot(2 \mu \varepsilon(u))+\nabla p & =f_{S T}+f, \\
\frac{\partial \rho}{\partial t}+\nabla \cdot(\rho u) & =0,
\end{aligned}
$$




$$
\rho=\left(\rho_{v}-\rho_{l}\right) H(\alpha)+\rho_{l},
$$

where $H$ is a Heaviside function. The mass conservation in the domain $\Omega$ reads

$$
\frac{\partial \rho}{\partial t}+\nabla \cdot(\rho u)=0
$$

94 We define a surface mass transfer rate $\dot{m}\left[\mathrm{~kg} \cdot \mathrm{m}^{-2} \cdot \mathrm{s}^{-1}\right]$ to quantify the exchange of mass between the liquid

$$
\nabla \cdot u=\dot{m}\left(\frac{1}{\rho_{v}}-\frac{1}{\rho_{l}}\right)|\nabla \alpha| \delta(\alpha) .
$$

where $\delta$ is a Dirac function. Expanding (11) and (12) leads to

$$
\begin{aligned}
\rho_{v} \frac{\partial H(\alpha)}{\partial t}+\rho_{v} H(\alpha) \nabla \cdot u+\rho_{v} u \cdot \nabla H(\alpha) & =\dot{m}|\nabla \alpha| \delta(\alpha), \\
-\rho_{l} \frac{\partial H(\alpha)}{\partial t}+\rho_{l}(1-H(\alpha)) \nabla \cdot u-\rho_{l} u \cdot \nabla H(\alpha) & =-\dot{m}|\nabla \alpha| \delta(\alpha) .
\end{aligned}
$$

By dividing (13) and (14) by their respective density and summing, we obtain the new mass conservation equation

The velocity is not divergence free since a mass transfer occurs at the interface between the vapor and the liquid.

Since a transfer of mass occurs, the level set equation will be modified to advect the level set not only from the velocity obtained through the resolution of the Navier-Stokes equations but also the velocity of the vapor front. This is obtained by summing (13) and (14) and dividing by $\left(\rho_{v}-\rho_{l}\right)$ :

$$
\frac{\partial H(\alpha)}{\partial t}+u \cdot \nabla H(\alpha)=\frac{\rho}{\rho_{l}-\rho_{v}} \nabla \cdot u
$$

108 Considering the derivative in time and in space of the Heaviside function, $\frac{\partial H(\alpha)}{\partial t}=\frac{\partial H(\alpha)}{\partial \alpha} \frac{\partial \alpha}{\partial t}=\delta(\alpha) \frac{\partial \alpha}{\partial t}$ and $109 \nabla H(\alpha)=\delta(\alpha) \nabla \alpha$, we obtain

$$
\delta(\alpha) \frac{\partial \alpha}{\partial t}+\delta(\alpha) u \cdot \nabla \alpha=\frac{\rho}{\rho_{l}-\rho_{v}} \nabla \cdot u
$$


110 In the case of a divergence free velocity fields, Eq (17) resumes to the standard level set equation. Replacing $111 \nabla \cdot u$ by $(15)$ leads to

$$
\delta(\alpha) \frac{\partial \alpha}{\partial t}+\delta(\alpha) u \cdot \nabla \alpha=\frac{\rho}{\rho_{l}-\rho_{v}} \dot{m}\left(\frac{1}{\rho_{v}}-\frac{1}{\rho_{l}}\right)|\nabla \alpha| \delta(\alpha) .
$$

112 Extending to the whole domain and simplifying leads to

$$
\frac{\partial \alpha}{\partial t}+u \cdot \nabla \alpha=\frac{\rho}{\rho_{l} \rho_{v}} \dot{m}|\nabla \alpha|
$$

The level set equation now reads

$$
\frac{\partial \alpha}{\partial t}+\left[u-\frac{\rho}{\rho_{l} \rho_{v}} \dot{m} \frac{\nabla \alpha}{|\nabla \alpha|}\right] \cdot \nabla \alpha=0
$$

The level set is now advected by the velocity $u$ obtained from the resolution of the Navier-Stokes and by 115 a velocity representing the vaporization of the water.

116 Similarly, one can use the same derivation for the energy equation. Neglecting the heat generated by 117 viscosity forces and capillary forces, the energy equation reads

$$
\rho c_{p}\left(\frac{\partial T}{\partial t}+u \cdot \nabla T\right)-\nabla \cdot(k \nabla T)=-\left(L+\left(c_{p}^{v}-c_{p}^{l}\right)\left(T-T_{\mathrm{sat}}\right)\right) \dot{m} \delta(\alpha)|\nabla \alpha| \frac{\rho^{2}}{\rho_{v} \rho_{l}} .
$$

where $T$ is the temperature, $T_{\text {sat }}$ is the sturation temperature, $c_{p}$ is the specific heat, $c_{p}^{v}\left(\right.$ resp. $\left.c p_{l}\right)$ is the specific heat in the vapor (resp. in the liquid) and $\mathrm{k}$ is the thermal conductivity. The formulation accounts naturally for the thickness of the interface. It is a generic formulation that requires the definition of the mass transfer rate $\dot{m}$.

Remark 1. The smoothing of the sharp mass flux presented in [37], using an averaged density is automatically obtained in (19). The origin of the smoothing is due to the smoothing of the distribution of the density across the interface.

\subsubsection{Derivation of the surface mass transfer rate}

The mass transfer rate is resolved using the heat released by an elementary volume $d V$ of liquid that has vaporized between the time $t$ and $t+d t$. We consider a domain composed of water and vapor (see Figure 1 ). The position of the interface at the time $t$ is given by $\alpha(t), d S$ and $n$ being respectively the corresponding elementary surface and its normal pointing in the vapor direction.

The heat released $d Q$ by a volume $d V$ of liquid that has vaporized during the elapsed time between $t$ and $t+d t$ is:

$$
d Q=\rho_{l} L d V
$$




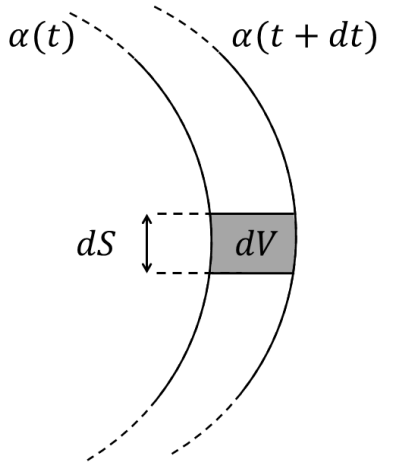

Figure 1: Volume $d V$ that has vaporized between $t$ and $t+d t$.

132 where $L$ is the enthalpy of vaporization $\left[\mathrm{J}_{\mathrm{kg}} \mathrm{kg}^{-1}\right]$. The heat fluxes are defined respectively in the vapor and the liquid by $\phi_{v}=-k_{v} \nabla T_{v}$ and $\phi_{l}=-k_{l} \nabla T_{l}$ where $k_{v}$ and $k_{l}$ are the thermal conductivity in the vapor 134 and the liquid respectively. Vaporization (resp. condensation) occurs when the jump of fluxes across the 135 interface $\llbracket \phi \rrbracket=\left[\phi_{v}-\phi_{l}\right]_{\mid \alpha=0} . n$ is positive (resp. negative). Then $d Q$ reads:

$$
d Q=\int_{t^{\prime}=t}^{t+d t} \llbracket \phi \rrbracket d S d t^{\prime}
$$

136 Differentiating and taking the limit for $d t \rightarrow 0$ leads to

$$
\lim _{d t \rightarrow 0} \rho_{l} L \frac{\alpha(t+d t)-\alpha(t)}{d t}=\lim _{d t \rightarrow 0} \frac{1}{d t} \int_{t^{\prime}=t}^{t+d t}\left(-k_{v} \nabla T_{v}+k_{l} \nabla T_{l}\right)_{\mid \alpha=0} . n d t^{\prime} .
$$

137 We obtain the surface mass transfer rate, the so-called Stefan condition,

$$
\dot{m}=\rho_{l} L \frac{d \alpha}{d t}=\left(-k_{v} \nabla T_{v}+k_{l} \nabla T_{l}\right)_{\mid \alpha=0} . n
$$

138 Eq. (25) requires the evaluation of the balance of fluxes at the interface $\left(-k_{v} \nabla T_{v}+k_{l} \nabla T_{l}\right)_{\mid \alpha=0} . n$. This 139 evaluation is not straightforward when using an implicit definition of the interface. We therefore choose 140 to use a delta formulation. Approximating the surface integral by mean of a delta Dirac function on each 141 elementary volume $\Omega_{i}$ containing the surface $S_{i}$ leads to

$$
\int_{S_{i}}\left(-k_{v} \nabla T_{v}+k_{l} \nabla T_{l}\right)_{\mid \alpha=0} \cdot n d S_{i}=\int_{\Omega_{i}} \delta(\alpha)\left(-k_{v} \nabla T_{v}+k_{l} \nabla T_{l}\right) \cdot n d \Omega_{i} .
$$

142 Then, integrating (25) over $\Omega_{i}$ gives an expression for the surface mass transfer $\dot{m}$ :

$$
\dot{m}=\frac{\int_{\Omega_{i}} \delta(\alpha)\left(-k_{v} \nabla T_{v}+k_{l} \nabla T_{l}\right) \cdot n d \Omega_{i}}{\int_{\Omega_{i}} \delta(\alpha) d \Omega_{i}}
$$




\subsection{Implicit surface tension}

A common way to introduce the surface tension in the Navier-Stokes equations is to use the Continuum Surface Force model [38]. It consists first in rewriting the surface force as a volume one as follows:

$$
f_{\mathrm{ST}}=-\gamma \kappa \delta \mathrm{n}
$$

where $\gamma$ is the surface tension coefficient, $\delta$ is a Dirac function locating the interface, $\kappa$ is the mean curvature and $n$ is the normal to the interface. Then (28) is used as a source term in the momentum equation (8). However, an explicit implementation imposes the following severe restriction on the time step [38]:

$$
\Delta t<(\Delta x)^{\frac{3}{2}} \sqrt{\frac{\rho_{l}+\rho_{v}}{4 \pi \gamma}} .
$$

149

If this time step restriction is not respected, spurious oscillations pollute the solution and destabilize the interface. However, this time step restriction penalizes the computational cost of numerical simulations since it is one or two orders of magnitude lower than the time step restriction of a CFL condition. To alleviate this restriction, we use the approach developed in [39] and widely used in [25, 40, 41]. Using differential geometry, the surface Laplacian $\Delta_{s} I_{\Gamma}$ of an identity mapping function is $-\kappa \mathrm{n}$. We apply the surface Laplacian operator $\Delta_{S}$ on $\mathrm{I}_{\Gamma}^{n+1}=\mathrm{I}_{\Gamma}^{n}+\mathrm{u}^{n+1} \Delta t$ which represents the evolution in time of an interface. The surface Laplacian can be decomposed into a standard Laplacian $\nabla^{2} u-\frac{\partial^{2} u}{\partial \mathrm{n}^{2}}-\kappa \frac{\partial u}{\partial \mathrm{n}}$. Multiplying by the surface tension coefficient leads to the new expression for the surface tension:

$$
f_{\mathrm{ST}}=-\gamma \kappa \delta \mathrm{n}-\gamma \delta \Delta t\left(\frac{\partial^{2} \mathrm{u}}{\partial \mathrm{n}^{2}}+\kappa \frac{\partial \mathrm{u}}{\partial \mathrm{n}}-\nabla^{2} \mathrm{u}^{n+1}\right) .
$$

The usual term $-\gamma \kappa \delta \mathrm{n}$ is now completed by additional terms proportional to the time step. These additional terms act as an isotropic diffusion minus a diffusion in the normal direction of the interface. More details are provided in [25].

\subsection{Variational MultiScale method for the Navier-Stokes equations}

Following the developments from previous sections, we generalize the Navier-Stokes equations, into: Find the velocity $u$ and the pressure $p$ such that:

$$
\begin{aligned}
\rho\left(\partial_{t} u+u \cdot \nabla u\right)-\nabla \cdot(2 \mu \varepsilon(u))+\nabla p & =f_{S T}+f, \\
\nabla \cdot u & =f_{c},
\end{aligned}
$$

where $\rho, \mu, f_{S T}, f$ and $f_{c}$ are the density, the viscosity, the surface tension force, additional source term of the momentum equation and the source term of the continuity equation respectively.

The stability of the discrete formulation of Navier-Stokes depends on appropriate compatibility restrictions on the choice of the finite element spaces for the velocity and the pressure. Standard Galerkin mixed 
elements with continuous equal order linear/linear interpolation is not a stable discretization and exhibits uncontrollable oscillations that pollute the solution. The Variational MultiScale method, proposed by Hughes $[42,43]$, offers a general framework that deals with different and new variant of mixed variational formulations [44-47]. We briefly recall here the main steps.

First, let us consider a decomposition of the velocity and the pressure fields into resolvable coarse-scale and unresolved fine-scale:

$$
\begin{aligned}
& u=u_{h}+\tilde{u}, \\
& p=p_{h}+\tilde{p},
\end{aligned}
$$

Likewise, we apply the same decomposition for the weighting functions. The unresolved fine-scales are usually modeled using residual based terms that are derived consistently. The static condensation consists in substituting the fine-scale solution into the large-scale problem providing additional terms, tuned by a local stabilizing parameter. The latter enhances the stability and accuracy of the standard Galerkin formulation.

Second, to derive the stabilized formulation, we solve the fine scale problem, defined on the sum of element interiors and written in terms of the time-dependent large-scale variables. Then we substitute in the coarse problem, the fine-scale solution approximated within each element by:

$$
\begin{aligned}
\tilde{u} & =\sum_{\mathcal{T}_{h}} \tau_{u} \tilde{P}_{u}\left(R_{u}\right), \\
\tilde{p} & =\sum_{\mathcal{T}_{c}} \tau_{c} \tilde{P}_{c}\left(R_{c}\right),
\end{aligned}
$$

where $R_{u}$ and $R_{c}$ are the finite element residuals. $\tilde{P}_{u}$ and $\tilde{P}_{p}$ are projection operators taken as the identity in this work. $\tau_{u}$ and $\tau_{c}$ are the so called stabilization parameters. Thus, we eliminate the explicit appearance of the fine-scale while still modeling their effects. More details are provided in [25, 29].

To simplify the notation, we use $f_{m}$ and $f_{c}$ as the source terms in (31)-(32), adding the explicit terms of (30) into $f_{m}$. Inserting the expression for the subscales, we finally obtain the stabilized finite element 92 problem. The new variational formulation reads for the coarse scale,

$$
\begin{cases}\left(\rho \partial_{t}\left(u_{h}+\tilde{u}\right), v_{h}\right)+\left(\rho\left(u_{h}+\tilde{u}\right) \cdot \nabla\left(u_{h}+\tilde{u}\right), v_{h}\right)-\left(p_{h}+\tilde{p}, \nabla \cdot v_{h}\right) & \\ +\left(2 \mu \varepsilon\left(u_{h}\right): \varepsilon\left(v_{h}\right)\right)+\left(\gamma \delta \Delta t \nabla\left(u_{h}+\tilde{u}\right): \nabla v_{h}\right)=\left(f_{m}, v_{h}\right) & \forall v_{h} \in V_{h, 0}, \\ \left(\nabla \cdot\left(u_{h}+\tilde{u}\right), q_{h}\right)=\left(f_{c}, q_{h}\right) & \forall q_{h} \in Q_{h},\end{cases}
$$

93 and for the fine scale, 


$$
\begin{cases}\left(\rho \partial_{t}\left(u_{h}+\tilde{u}\right), \tilde{v}\right)+\left(\rho\left(u_{h}+\tilde{u}\right) \cdot \nabla\left(u_{h}+\tilde{u}\right), \tilde{v}\right)-\left(p_{h}+\tilde{p}, \nabla \cdot \tilde{v}\right) & \\ +(2 \mu \varepsilon(\tilde{u}): \varepsilon(\tilde{v}))+\left(\gamma \delta \Delta t \nabla\left(u_{h}+\tilde{u}\right): \nabla \tilde{v}\right)=\left(f_{m}, \tilde{v}\right) & \forall \tilde{v} \in \tilde{V}, \\ \left(\nabla \cdot\left(u_{h}+\tilde{u}\right), \tilde{q}\right)=\left(f_{c}, \tilde{q}\right) & \forall \tilde{q} \in \tilde{Q} .\end{cases}
$$

where $R_{u}$ and $R_{c}$ are the residuals defined by

$$
\begin{aligned}
& R_{u}=f_{m}-\rho \partial_{t} u_{h}-\rho u_{h}^{c} \cdot \nabla u_{h}-\nabla p_{h}, \\
& R_{c}=f_{c}-\nabla \cdot u_{h} .
\end{aligned}
$$

At this level, two assumptions can be made to simplify the resolution of the fine scale equation: the subscales are considered quasi-static and the convection is approximated by $\left(u_{h}+\tilde{u}\right) \cdot \nabla\left(u_{h}+\tilde{u}\right) \approx u_{h}^{c} \cdot \nabla\left(u_{h}+\tilde{u}\right)$. Therefore, by formulating the expression of $\tilde{u}$ and $\tilde{p}$ by substituting them into the large-scales equation, and applying integration by parts, the system to solve becomes finally

$$
\left\{\begin{array}{lr}
\left(\rho \partial_{t} u_{h}, v_{h}\right)+\left(\rho u_{h}^{c} \cdot \nabla u_{h}, v_{h}\right)-\left(p_{h}, \nabla \cdot v_{h}\right)+\left(2 \mu \varepsilon\left(u_{h}\right): \varepsilon\left(v_{h}\right)\right)+\left(\gamma \delta \Delta t \nabla u_{h}: \nabla v_{h}\right) \\
-\sum_{K \in \mathcal{T}_{h}}\left(\tau_{u} R_{u}, \rho u_{h}^{c} \cdot \nabla v_{h}\right)-\sum_{K \in \mathcal{T}_{h}}\left(\tau_{c} R_{c}, \nabla \cdot v_{h}\right)=\left(f_{m}, v_{h}\right) & \forall v_{h} \in V_{h, 0}, \\
\left(\nabla \cdot u_{h}, q_{h}\right)-\sum_{K \in \mathcal{T}_{h}}\left(\tau_{u} R_{u}, \nabla q_{h}\right)=\left(f_{c}, q_{h}\right) & \forall q_{h} \in Q_{h},
\end{array}\right.
$$

Note that in the case of strongly anisotropic meshes with highly stretched elements, the definition of the stabilization parameters is still an open problem and plays a critical role in the design of the stabilizing coefficients. In [25] the authors propose a particular choice of the stabilizing parameters suitable for anisotropic partitions that we adopt here.

By comparing the standard Galerkin method with the proposed stable formulation, additional integrals that are evaluated element-wise are involved. These additional terms, obtained by replacing the approximated $\tilde{u}$ and $\tilde{p}$ into the large-scale equation, represent the effects of the sub-grid scales and above all take into account the modified surface tension terms. They are introduced in a consistent way to the Galerkin formulation and enable to overcome the instability of the standard formulation arising in convection dominated flows and to deal with the pressure instabilities [48].

\section{Edge-based mesh adaptation for multiphase flows}

Anisotropic mesh adaptation on unstructured meshes plays an important role in the efficient numerical simulation of multiphase flow problems since it helps keeping high accuracy while reducing the dedicated CPU time to these simulations $[49,50]$. As it is shown in [25] and more recently in [51], anisotropic mesh adaptation outperforms adaptive octree. Small features are captured more easily using anisotropic mesh 
than using octree. The use of anisotropic mesh adaptation enables to reduce the number of integration points

\section{Figure 2.}

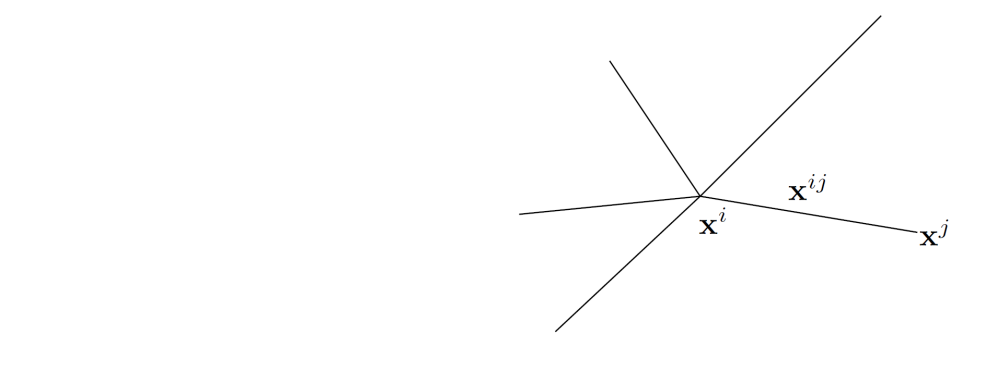

Figure 2: Patch associated with node $x^{i}$ by one order of magnitude [51]. The idea is to concentrate elements in regions where the solution exhibits a rapid variation of either the solution or its derivatives. In this case, it would be the large temperature gradients, the large jumps at the interfaces as well as the change in direction for the velocity fields. The objective is then to get the smallest error possible while controlling the number of mesh nodes. A vector or a scalar monitor function is used in order to control the size, shape and orientation of the elements of the mesh to be generated. This function is usually designed to give an estimate of some measure of the solution error which is then equidistributed over each mesh cell. The mesh adaptation algorithm is built in order to compute a mesh and a numerical solution. At each stage, we compute a numerical solution on the current mesh and we evaluate an estimation of the interpolation error. We set up a minimization problem that aims at minimizing the interpolation error in the $L_{1}$-norm, independently of the problem at hand [32]. To take into account the solution development, we derive an optimal metric that minimizes its interpolation error. Therefore, a new mesh is generated according to this metric field. The originality in the approach lies also in its simplicity to compute the metric and the associated edge-based error estimator developed below.

\subsection{Edge-based error estimation}

Let $u_{h}$ be a P1 finite element approximation obtained by applying the Lagrange interpolation operator to a regular function $u \in C^{2}(\Omega)$. At each vertex $i$ of the mesh, we have $U_{i}=u\left(x^{i}\right)=u_{h}\left(x^{i}\right)$ (where $x^{i}$ are the coordinates of the vertex $i$ ). Let $\Gamma(i)$ be the "patch" associated to a vertex $x_{i}$ of the mesh defined as the set of nodes which share one edge with $x_{i}$, and let us denote by $x_{i j}$ the edge connecting $x_{i}$ to $x_{j}$ as in

(1)

34 The gradient $\nabla u^{h} \cdot x^{i j}$ on the edge $x_{i j}$ is continuous, therefore we can write

$$
U^{j}=U^{i}+\nabla u^{h} \cdot x^{i j},
$$

which leads to

$$
\nabla u_{h} \cdot x^{i j}=U^{j}-U^{i}
$$




$$
G^{i}=\left(X^{i}\right)^{-1} \sum_{j \in \Gamma(i)} U^{i j} x^{i j}
$$

The work performed in [32] allows us to write the following error estimator:

with $H_{u}$ the Hessian of $u$. At the node $x^{i}$, we seek the recovered gradient $g^{i}$ of $u_{h}$

$$
\nabla g_{h} \cdot x^{i j}=g^{j}-g^{i}
$$

$$
\begin{array}{r}
\left(\nabla g_{h} \cdot x^{i j}\right) \cdot x^{i j}=\left(g^{j}-g^{i}\right) \cdot x^{i j} \\
\left(H_{u} \cdot x^{i j}\right) \cdot x^{i j}=g^{i j} \cdot x^{i j}
\end{array}
$$
suitable to define an error indicator function associated to the edge $x^{i j}$ as

$$
e^{i j}=\left|g^{i j} \cdot x^{i j}\right|
$$

\subsection{Gradient recovery procedure}

The gradient recovery procedure relies on the following optimization problem:

$$
G^{i}=\arg \min _{G}\left(\sum_{j \in \Gamma(i)}\left|\left(G-\nabla u_{h}\right) \cdot x^{i j}\right|^{2}\right),
$$
$X^{i}$ the length distribution tensor at node $i$

$$
X^{i}=\frac{1}{|\Gamma(i)|}\left(\sum_{j \in \Gamma(i)} x^{i j} \otimes x^{i j}\right),
$$
the recovered gradient $G^{i}$ in terms of the length distribution tensor

Therefore, the estimated error $e_{i j}$ is thus written as

$$
e_{i j}=G^{i j} \cdot x^{i j}
$$

$$
\left\|\nabla u^{h} \cdot x^{i j}-\nabla u\left(x^{i}\right) \cdot x^{i j}\right\| \leq \max _{y \in\left|x^{i}, x^{j}\right|}\left|x^{i j} \cdot H_{u}(y) \cdot x^{i j}\right|,
$$

We want the projection of the Hessian based on the gradient at the extremities of the edge, thus we have

with $g^{i j}=g^{j}-g^{i}$. It can be shown [32] that the quantity $\left|g^{i j} \cdot x^{i j}\right|$ gives a second order accurate approximation of the second derivative of $u$ along the edge $x^{i j}$. Motivated by the fact that, for P1 finite elements on anisotropic meshes, edge residuals dominate a posteriori errors, as proved in [52], it is therefore

And this error, is the exact interpolation error along the edge and allows to evaluate the global $L_{1}$ error. However, the gradient is not know at the vertices, thus a recovery procedure has to be considered.

where $G^{i}$ is the recovered gradient. Denoting by $\otimes$ the tensor product between two vectors, let us introduce

whose purpose is to give an average representation of the distribution of edges in the patch. Let us express 


\subsection{Metric construction}

In order to relate the error indicator defined in (51) to a metric suitable for a mesh adaptation procedure, we introduce the concept of stretching factor $s^{i j}$ defined as the ratio between the length of the edges $x^{i j}$ after the adaptation procedure and after the adaptation procedure, [32]. The metric takes the following expression:

$$
\widetilde{M}^{i}=\left(\widetilde{X}^{i}\right)^{-1}
$$

where $\widetilde{X}^{i}$ is defined as:

$$
\tilde{X}^{i}=\frac{1}{|\Gamma(i)|}\left(\sum_{j \in \Gamma(i)} s^{i j} \otimes s^{i j}\right) .
$$

271 The stretching factor $s^{i j}$ of the edge $i j$ is chosen so that the total number of nodes in the mesh is kept fixed 272 and is defined as

$$
s^{i j}=\left(\frac{e_{i j}}{e(N)}\right)
$$

274 where $e(N)$ is the total error .

\subsection{Mesh adaption criteria}

In multiphase applications, the material interface between liquid and gas need to be modeled accurately. Two strategies are commonly used; we refer to them in this work as explicit and implicit adaptation. In the first one, we design and pre-adapt the mesh around the boundaries and in some regions of interest. The obtained adapted mesh will be used all along the simulation, provided that the flow exhibits a bounded level of unsteadiness. The criteria for the mesh adaptation are geometric and do not depend on the solution. Whereas, the implicit strategy imposes a dynamic mesh adaptation that changes the mesh frequently and minimizes as possible the prescribed error. Consequently, it requires a criterion based solely on the solution.

The common way to adapt a mesh to several variables, such as the velocity and the level set function, is to compute the metrics corresponding to each of them and then to produce a unique metric by an operation known as the intersection of metrics. In this work, we simplify this operation and we use one metric that account for different variables. Therefore, based on the theory proposed in the previous section, it is possible to extend definition (47) to account for several sources of error. In the following numerical experiments, the adaptivity accounts for the velocity components $V_{k}$, its magnitude $|V|$, the level set function $\alpha$ and the temperature $T$, by defining the following vector of sources of error:

$$
\mathbf{v}\left(\mathbf{x}^{i}\right)=\left\{\frac{V_{k}^{i}}{\left|V^{i}\right|}, \frac{\left|V^{i}\right|}{\max _{j}\left|V^{j}\right|}, \frac{\alpha^{i}}{\max (\alpha)}, \frac{T^{i}}{\max (T)}\right\}
$$


291 the velocity norm $|V|$, the temperature and the level set function $\alpha$ by their respective global maximum), a

292 field that is much larger in absolute value does not dominate the error estimator, and the variations of all

293 variables are fairly taken into account. is finer with an anisotropic adaptive mesh.
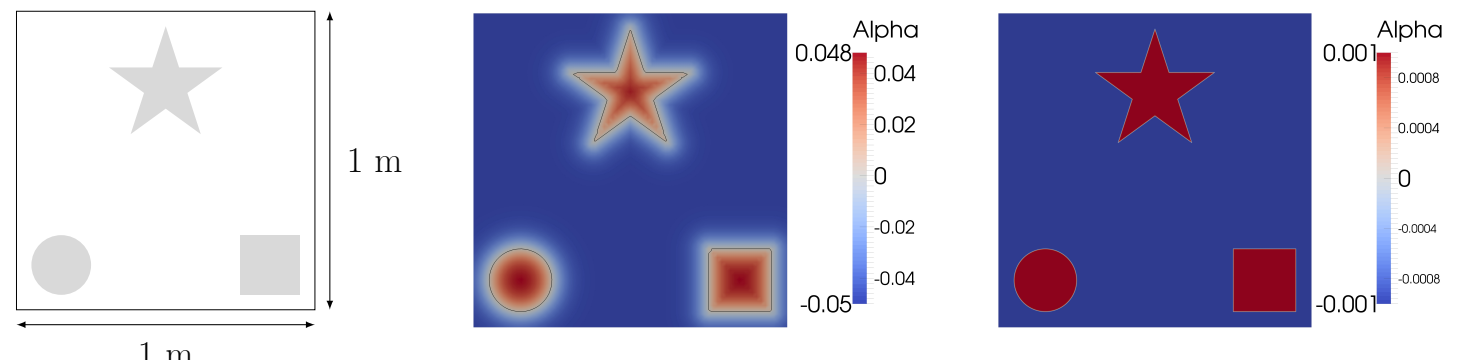

Figure 3: Three immersed objects inside a squared cavity (left). Filtered level set function for 10,000 elements in a structured mesh (middle) and in an adaptive mesh (right). The use of an adaptive mesh enables a finer interface transition. Respective meshes are shown in Figure 5.

306

307

308

309

310 311 This yields a great reduction of the number of triangles. These results give confidence that the proposed 312 framework allows to deal with different shapes, with angles, singular point and curvatures.

We aim to show the flexibility of the proposed mesh adaptation technique to deal with multiphase flows. Therefore we consider three fixed objects defined by level set functions inside a squared domain (see Figure 3 ). The circle of radius $0.1 \mathrm{~m}$ is centered at $(0.15 ; 0.15)$. The square of $0.20 \mathrm{~m}$ size is centered at $(0.85 ; 0.15)$. The regular pentagram is centered at $(0.5 ; 0.75)$ and the radius of the circumcircle is $0.2 \mathrm{~m}$. We choose to position them close to the wall to assess the capacity of the method to capture the features of the geometry close to a boundary.

In multiphase simulations, we use the level set function to define the properties in each phase. The physical properties are usually discontinuous across the interface. To avoid discontinuities which lead to numerical errors, we use a smooth Heaviside function computed from the level set function. This creates an interface transition with a thickness of few elements. The use of mesh adaptation techniques enables to reduce this thickness. As it is depicted in Figure 3, for a given number of elements $(10,000)$, the transition

Figure 4 shows the obtained zero isovalue of the level set functions using different number of nodes. The comparison with structured meshes using the same number of nodes shows that anisotropic mesh adaptation allows easily to keep very good accuracy of the geometry, even for a low number of nodes. Figure 5 shows the correct orientation and deformation of the mesh elements (longest edges parallel to the boundary). 
314 perimeter and the total area of the three immersed objects and we plot the error between the analytical

315 and the numerical solutions. We also plot the error for a structured mesh. Figure 6 confirms the advantage

316 of using anisotropic adaptive meshes for multiphase flows. For a given accuracy, at least ten times more elements are required in a structured mesh.

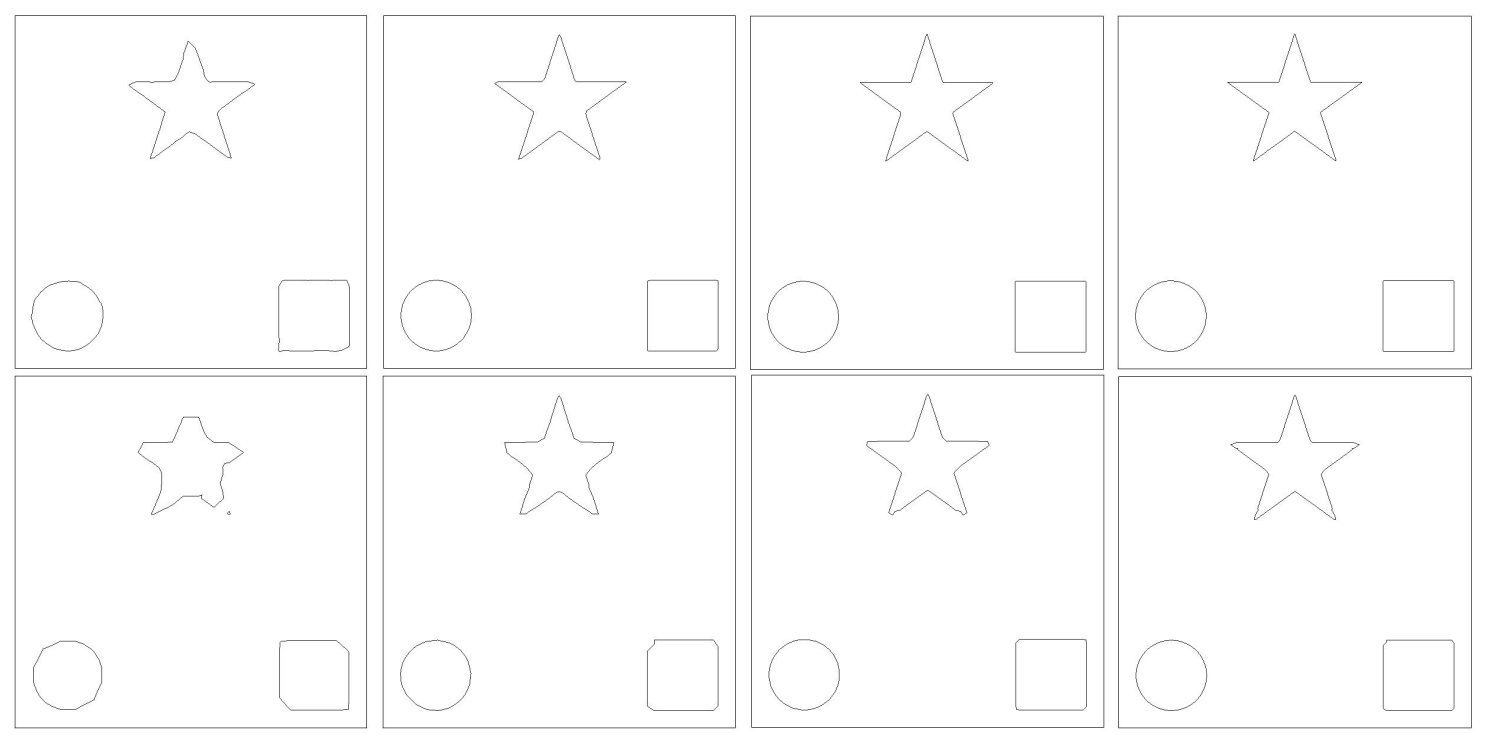

Figure 4: Zero isovalue of the level set function for 1,000, 2,000, 5,000 and 10,000 elements. First line: result with adaptive meshing. Second line: result with structured meshes.
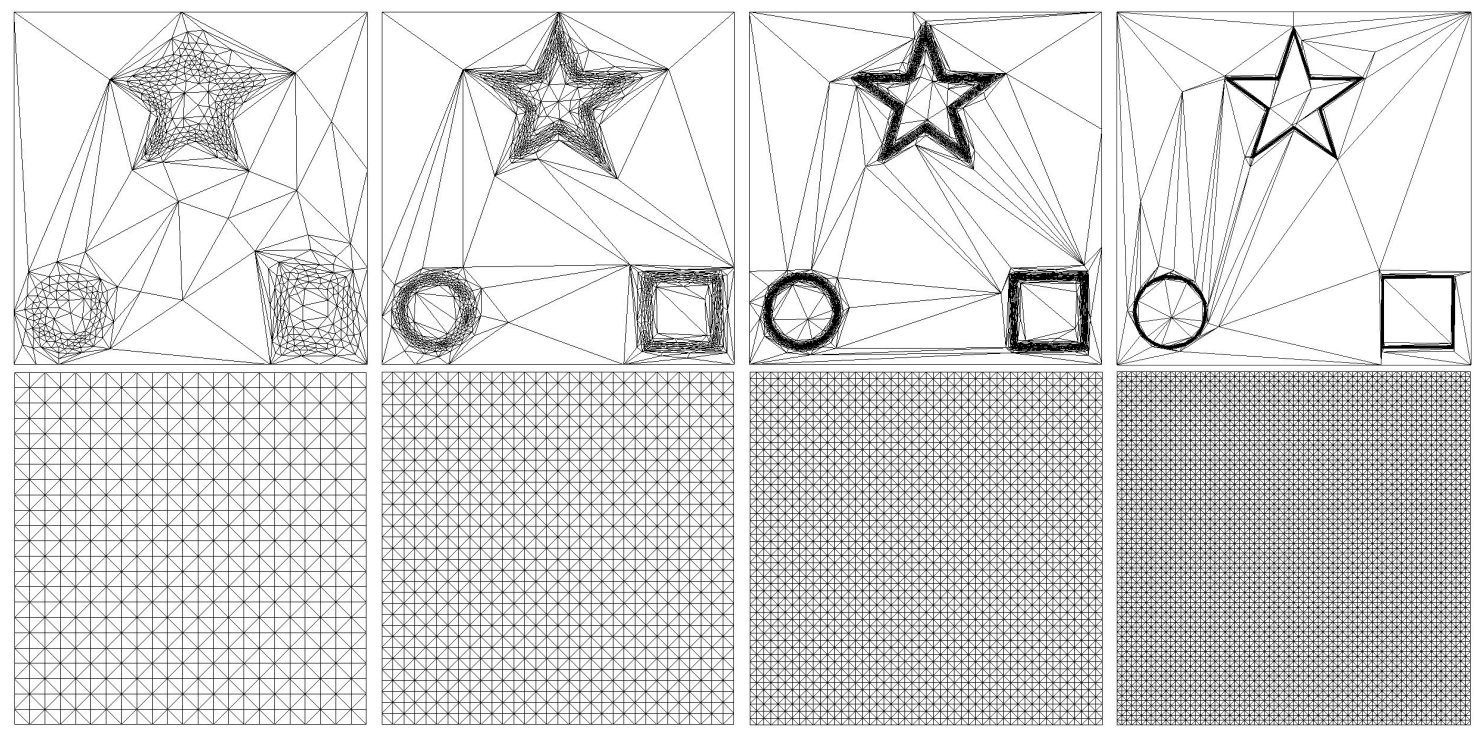

Figure 5: The obtained mesh for 1,000, 2,000, 5,000 and 10,000 elements. 

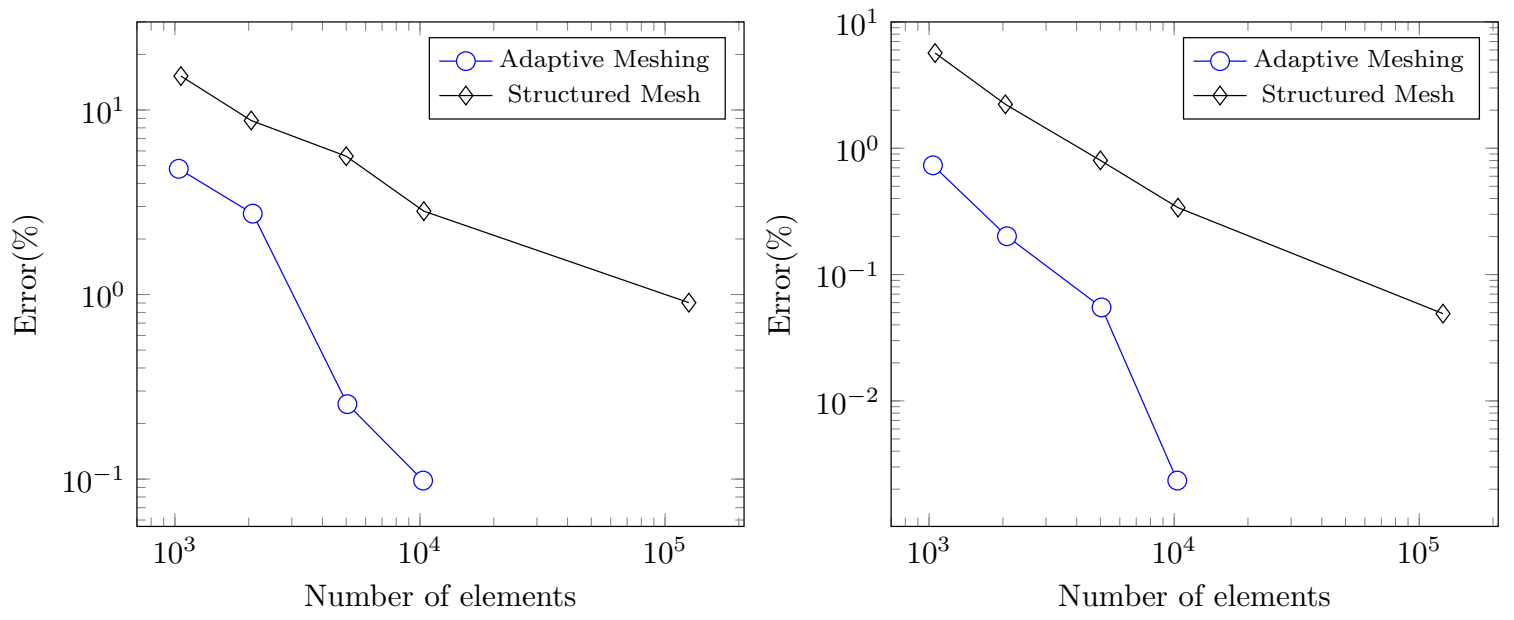

Figure 6: Percentage of error for the computation of the perimeter (left) and the area (right).

\section{Numerical examples}

The proposed adaptive Eulerian framework for the simulation of both boiling and evaporation phenomena is verified and validated in this section through a variety of test problems. Throughout this section, all the obtained results will be compared to either analytical or numerical available solutions. In the last test case, we bring forward a new problem with the experimental data on the cooling of a 3D heated solid inside a water tank.

\subsection{Stefan problem}

First, we consider the one-dimensional Stefan benchmark. It is a well known problem and serves as a benchmark to assess the accuracy of phase change models $[8,11,53]$. It is defined schematically in Figure 7. The domain is initially filled with water. The wall temperature is set constant and larger than the saturation temperature. The water is at saturation temperature. At $t>0$, a phase change occurs and induces a motion of the interface between the vapor and the water. The convective term in the energy conservation equation is neglected in both phases.

The position of the interface is given by

$$
s(t)=2 \chi \sqrt{\alpha_{v} t},
$$

332 where $\alpha_{v}$ is the thermal diffusivity defined by $\alpha=k_{v} /\left(\rho_{v} c_{p}^{v}\right)$ and $\chi$ is the solution of the transcendental 333 equation

$$
\frac{T_{\mathrm{sat}}-T_{\mathrm{wall}}}{\sqrt{\pi} L} c_{p}^{v}=\chi \operatorname{erf}(\chi) \exp \left(\chi^{2}\right)
$$




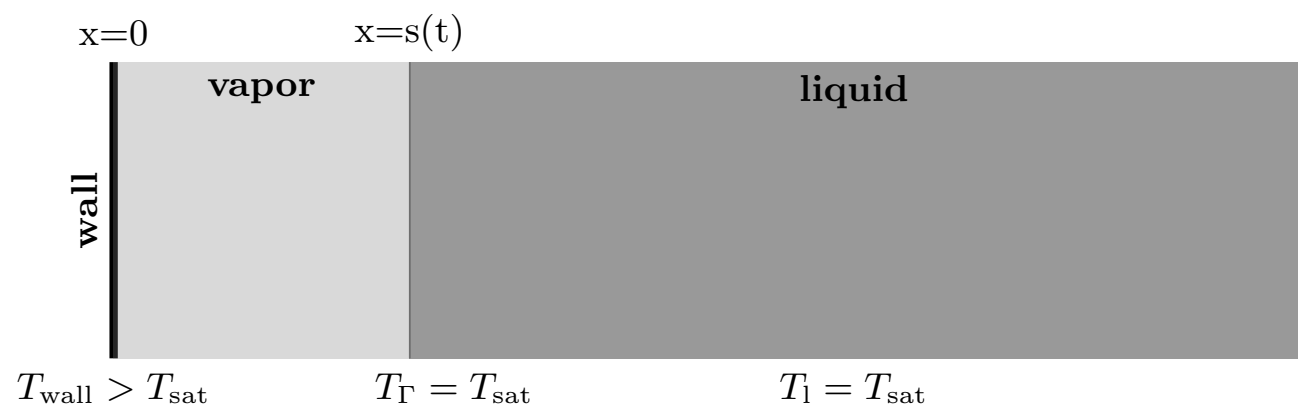

Figure 7: Initial setup for the classic Stefan problem.

334 derived in the case of a constant temperature in the liquid. The temperature in the vapor at a given time $t$ 335 is given by

$$
T(x, t)=T_{\text {wall }}+\frac{T_{\text {sat }}-T_{\text {wall }}}{\operatorname{erf}(\chi)} \operatorname{erf}\left(\frac{x}{2 \sqrt{\alpha_{v} t}}\right)
$$

336 We consider the physical properties for the water and the vapor given in Table 1 and we consider $T_{\text {wall }}-T_{\text {sat }}=$ 10K. We solve the transcendental equation using a Newton algorithm to find the value of $\chi$.

Table 1: Density, dynamic viscosity, specific heat and thermal conductivity for the vapor and the water at atmospheric pressure

\begin{tabular}{cccccc} 
& $\rho\left[\mathrm{kg} / \mathrm{m}^{3}\right]$ & $\mu[\mathrm{Pa} \cdot \mathrm{s}]$ & $c_{p}[\mathrm{~J} /(\mathrm{kg} \cdot \mathrm{K})]$ & $\mathrm{k}[\mathrm{W} /(\mathrm{m} \cdot \mathrm{K})]$ & $\mathrm{L}_{\mathrm{sat}}[\mathrm{J} / \mathrm{kg}]$ \\
\hline Vapor & 0.597 & $1.26 \times 10^{-5}$ & 2030 & 0.025 & \\
Water & 958.4 & $2.8 \times 10^{-4}$ & 4216 & 0.679 & $2.26 \times 10^{6}$ \\
\hline \hline
\end{tabular}

Figure 8 shows the evolution of the position of the interface for both the analytical and the numerical solution having good agreement. Figure 9 shows that the use of a delta Dirac function to compute the mass transfer rate results in a better convergence. This comparison was done by prescribing a maximum number of iterations (3000) to reach a residual $\left(10^{-7}\right)$ using the GMRES method for the resolution of the linear system. 


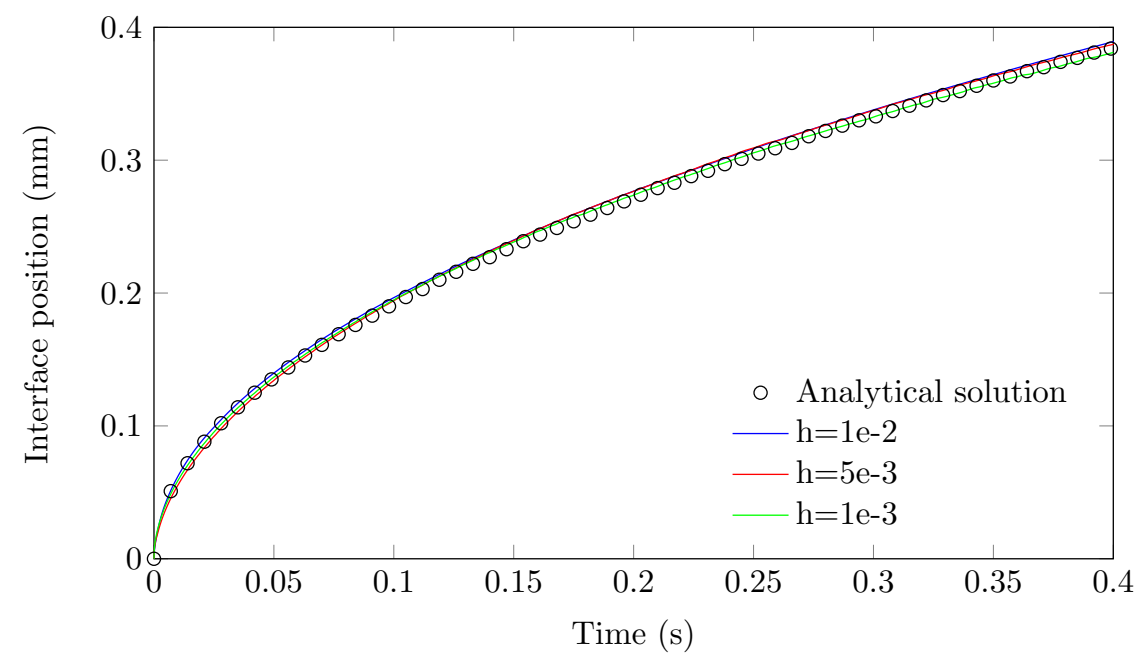

Figure 8: Evolution of the interface position for the Stefan problem.

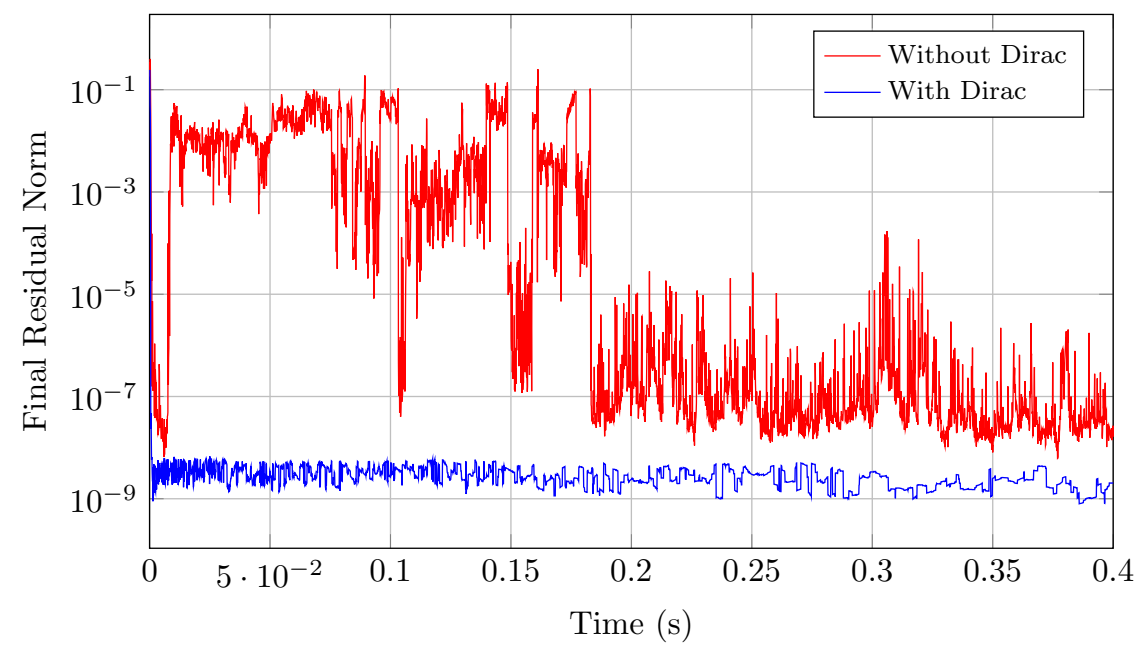

Figure 9: Final residual norm for the level set solver. 
This test case serves as a validation for the phase change model in several articles $[1,8,14,54]$. Indeed, 345 when the temperature at the wall is much larger than the saturation temperature, a persistent layer of 346 vapor forms and remains between the wall and the water. This regime is known as film boiling. In the 347 configuration depicted in Figure 10, a Rayleigh-Taylor instability is triggered due to the low density fluid 348 below the high density fluid. Furthermore, the phase change will induce a growth of the film, amplifying 349 the instability.

For a Rayleigh Taylor instability, the most unstable Taylor wavelength is, in 2D,

$$
\lambda_{0}=2 \pi\left(\frac{3 \gamma}{\left(\rho_{l}-\rho_{g}\right) g}\right)^{1 / 2}
$$

where $\gamma$ is the surface tension.

The physical parameters taken from [8] are given in Table 2. For this set of parameters, the most unstable wavelength is about $\lambda_{0} \approx 0.078 \mathrm{~m}$. Figure 10 shows the initial profile of the interface, defined by the following function:

$$
y=0.5+0.4 \cos \left(2 \pi x / \lambda_{0}\right)
$$

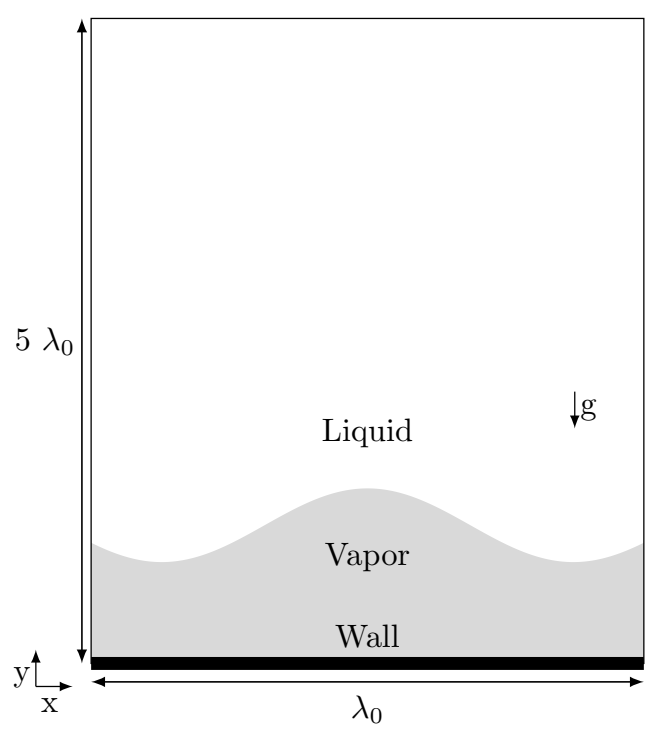

Figure 10: Setup for the 2D film boiling

Two cases will be studied. For the first case, the temperature at the wall is maintained constant at a temperature of $5 \mathrm{~K}$ above the saturation temperature and for the second, $10 \mathrm{~K}$. To assess the accuracy of the computations, the authors in $[1,8,54]$ used space-averaged Nusselt number obtained from the numerical 
simulation and compare it to correlation found in the literature. The local Nusselt number defined as the dimensionless heat flux through the wall is

$$
N_{\mathrm{u}}=\left.\frac{\lambda_{0}}{T_{w}-T_{\mathrm{sat}}} \frac{\partial T}{\partial y}\right|_{y=0} .
$$

Regarding the correlation of the Nusselt number in the literature, we use the correlation of Berenson [55] and Klimenko [56] given by

$$
N u_{B}=0.425(\operatorname{GrPr} / J a)^{1 / 4}
$$

361 and

$$
N u_{K}=0.1691(G r P r / J a)^{1 / 3} \text { for } J a<0.71 \text {. }
$$

where the Grashof number $G r=\rho_{v}\left(\rho_{l}-\rho_{v}\right) g \lambda_{0} / \mu_{v}^{2}$ represents the ratio of the buoyancy force over the viscous force, the Prandtl number $\operatorname{Pr}=\mu_{v} c_{p}^{v} / k_{v}$ represents the ratio of the momentum diffusivity over the thermal diffusivity and the Jakob number $J a=c_{p}^{v}\left(T_{w}-T_{\text {sat }}\right)$ the ratio of sensible heat over latent heat.

Table 2: Density, dynamic viscosity, specific heat and thermal conductivity for the vapor and the water at atmospheric pressure

\begin{tabular}{cccccc} 
& $\rho\left[\mathrm{kg} / \mathrm{m}^{3}\right]$ & $\mu[\mathrm{Pa} \cdot \mathrm{s}]$ & $c_{p}[\mathrm{~J} /(\mathrm{kg} \cdot \mathrm{K})]$ & $\mathrm{k}[\mathrm{W} /(\mathrm{m} \cdot \mathrm{K})]$ & $\mathrm{L}_{\mathrm{sat}}[\mathrm{J} / \mathrm{kg}]$ \\
\hline Vapor & 5.0 & 0.005 & 200 & 1.0 & \\
Water & 200 & 0.1 & 400 & 40 & $10^{4}$ \\
\hline \hline
\end{tabular}

Figure 11 shows the evolution of the temperature field and the interface for $\Delta T=10 K$. The vapor film grows to a mushroom shape due to the Rayleigh-Taylor instability. Since we performed 2D computations, no break up occurs due to surface tension. Therefore the mushroom cap rises along the channel followed by a long and thin filament. Notice that due to the width of the channel, there is clearly an effect of the lateral confinement on the shape of the vapor mushroom. Figure 12 shows the evolution of the mesh. The mesh is adapted using the anisotropic mesh adaptation procedure presented in this paper, using only 25,000 elements. Therefore the mesh is very fine at the vapor/water interface and at the bottom when the thermal gradient is the largest. The mesh remains coarse far from the interface, where the thermal gradient is null and the velocity field is still 0 .

The evolution of the space-averaged Nusselt number is depicted in Figure 13. The comparison with the correlation of Berenson and Klimenko shows a good agreement for both cases. 

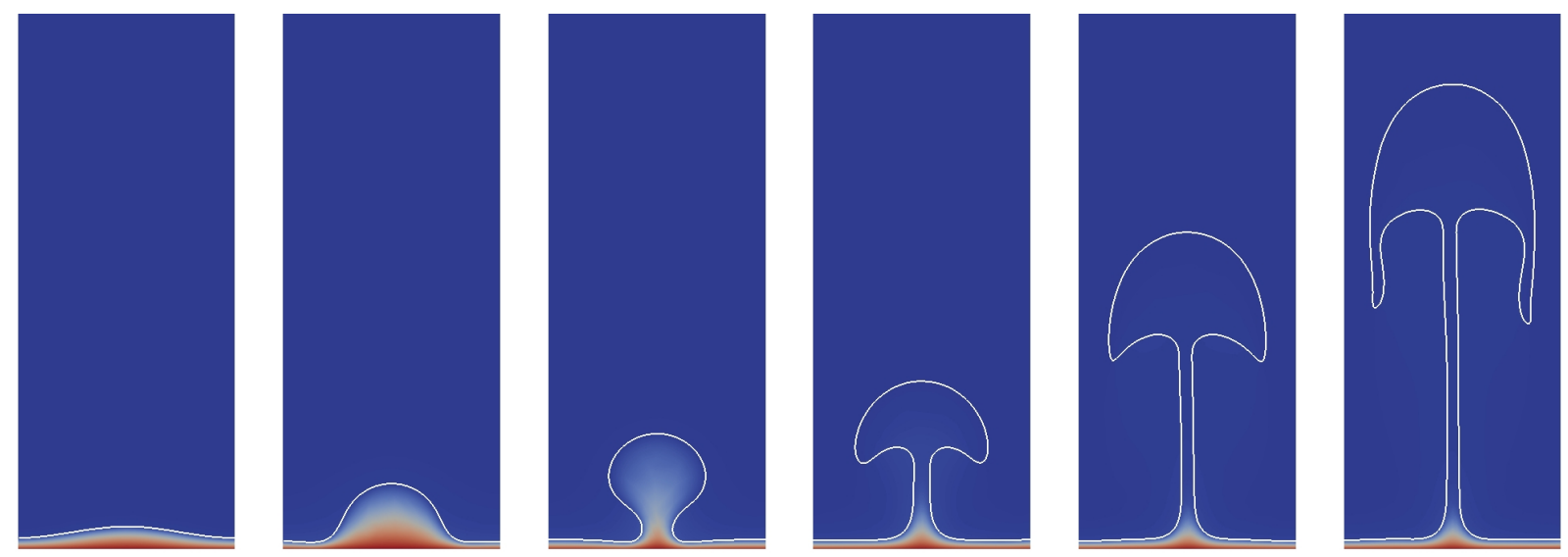

Figure 11: 2D Film boiling for $\Delta T=10 K$. Temperature field and interface location at $\mathrm{t}=0.01,0.2,0.3,0.4,0.7$ and $1.0 \mathrm{~s}$. The interface is represented by the white line.
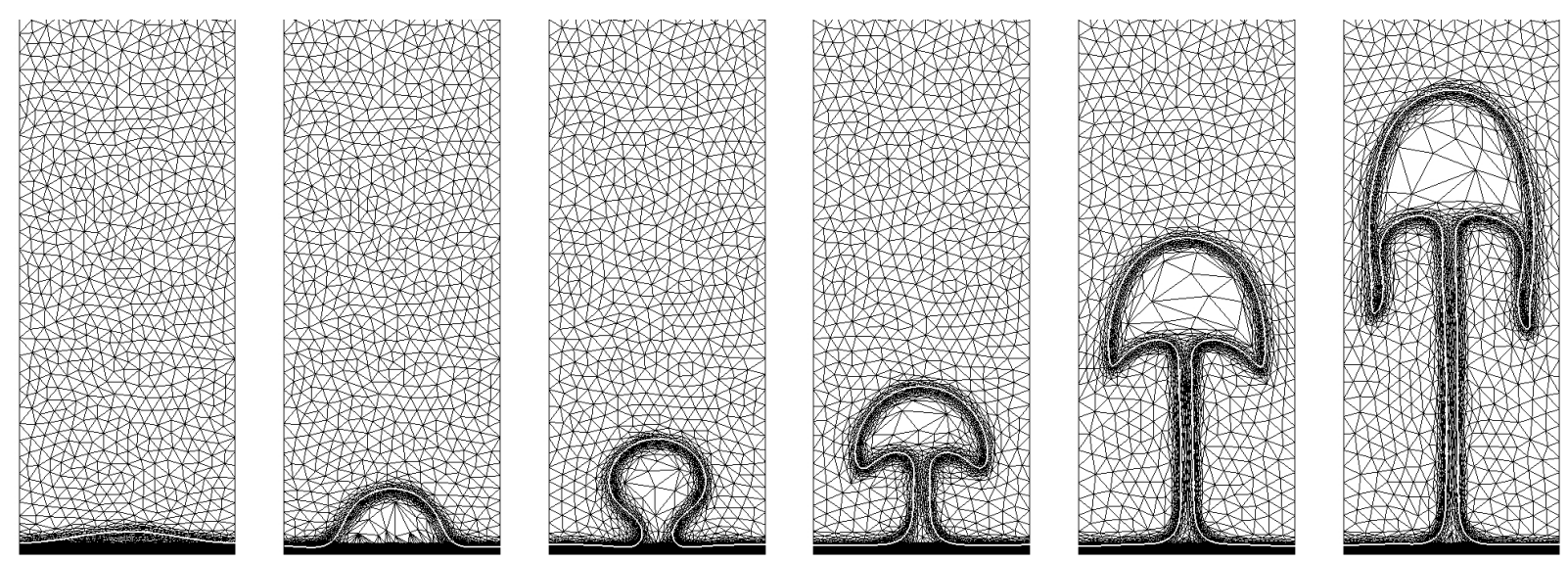

Figure 12: $2 \mathrm{D}$ Film boiling for $\Delta T=10 \mathrm{~K}$. Evolution of the mesh and the interface location at $\mathrm{t}=0.01,0.2,0.3,0.4,0.7$ and 1.0s. The interface is represented by the white line. 

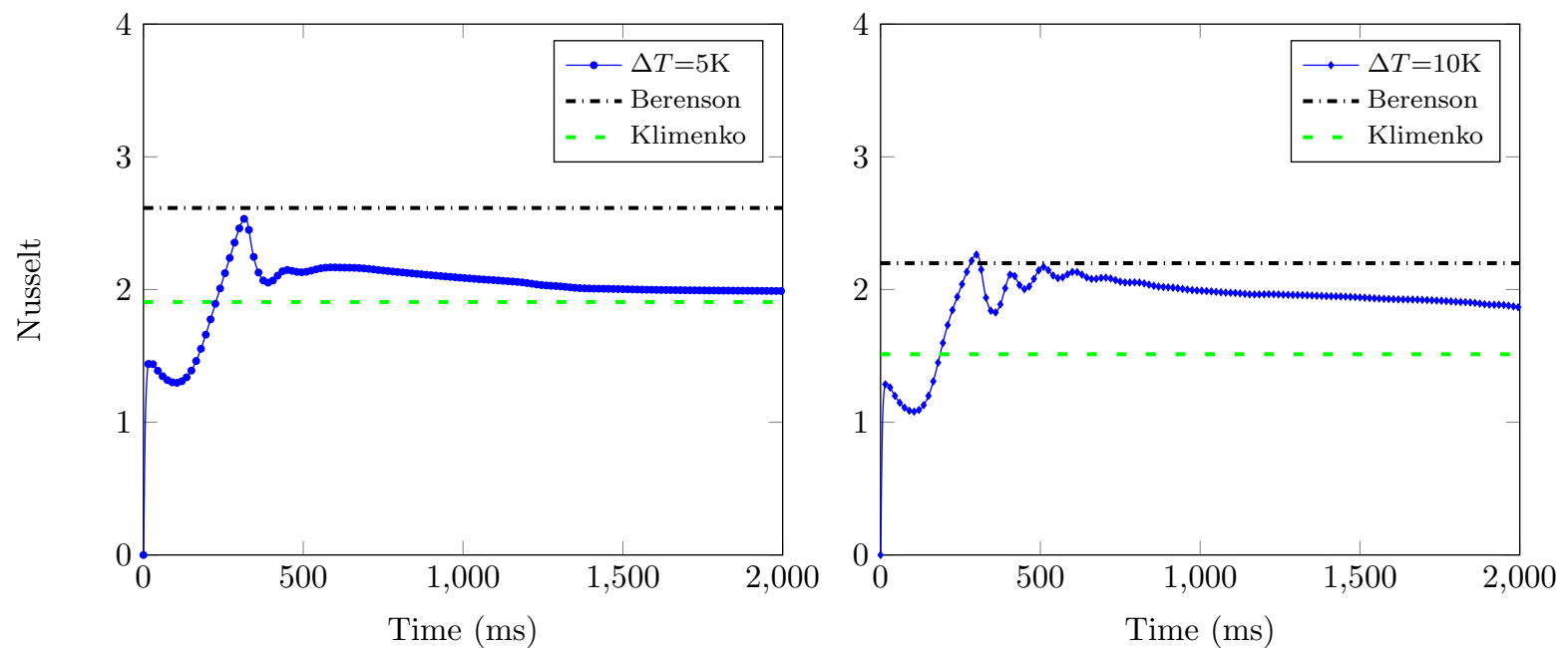

Figure 13: Evolution of the space-averaged Nusselt number for $\Delta T=5 K$ (left) and $\Delta T=10 K$ (right). 


\section{3. $3 D$ boiling - sensivitity analysis}

Several studies show that quench severity is dependent on different parameters: orientation, position, shape and size, agitation rate, fluid viscosity, and other variables. In this section, we will analyze at least the first three parameters. The objective is to show that the physical phenomena taking place are rich and that the experimental investigation remains generally limited. Indeed, the latter suffers from systematic revalidation of heat transfer coefficients when dealing with each parameter.

We consider then a water tank of dimension $L \times L \times L$ (see Figure 14), filled to three quarters. Two geometries are proposed; a hot metallic cylinder of length $0.5 \mathrm{~m}$ and diameter $0.1 \mathrm{~m}$ with $\mathrm{L}=1 \mathrm{~m}$ and a hot hollow cylinder (see Figure 18) of inner radius $3 \mathrm{~cm}$ and outer radius of $6 \mathrm{~cm}$ with $\mathrm{L}=0.40 \mathrm{~m}$.

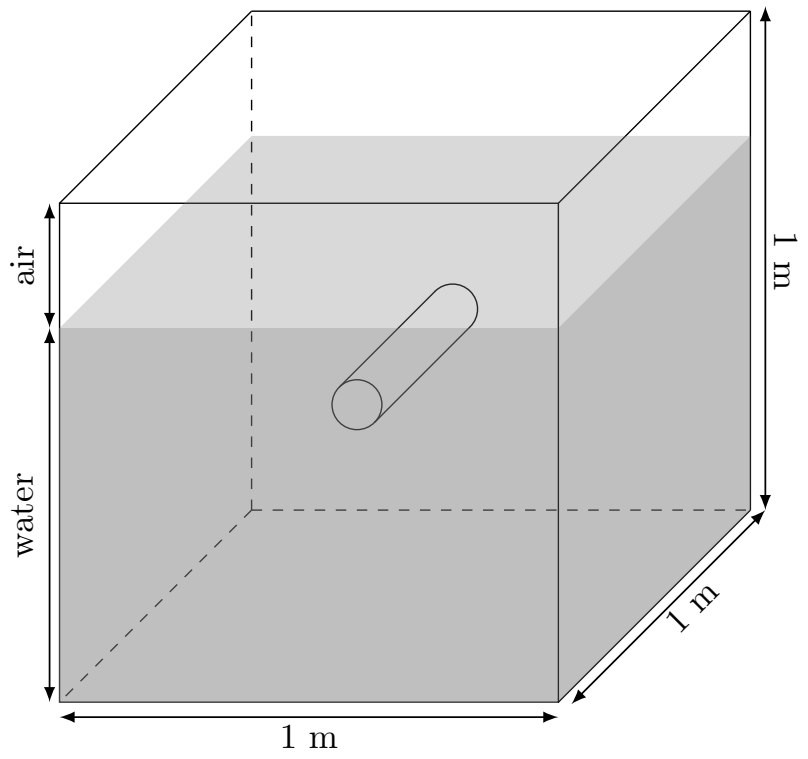

Figure 14: Set-up for the 3D film boiling

For the first geometry, three different configurations are considered. The cylinder is immersed horizontally at mid-height (see Figure 15), at a quarter of the height of the tank (see Figure 16) or vertically (see Figure 17).

Figure 11 shows a persistent vapor film surrounds the cylinder. As expected, the position of the cylinder has an important effect on the film evolution and thus on the cooling velocity of the cylinder. Furthermore, such distance from the cylinder to the free surface has a direct impact on the overall flow inside the quenching bath. The higher the distance, the higher the velocity of the vapor phase breaking up at the surface.

Whereas, when the cylinder is immersed vertically inside the water tank, the shape of the film is totally different. Indeed, we can notice in Figure 12 a periodic release of the film all along the surface. Thus, the distribution of the flow is more concentrated at the top of the cylinder. This induces lower cooling velocity when compared to the previous case. 

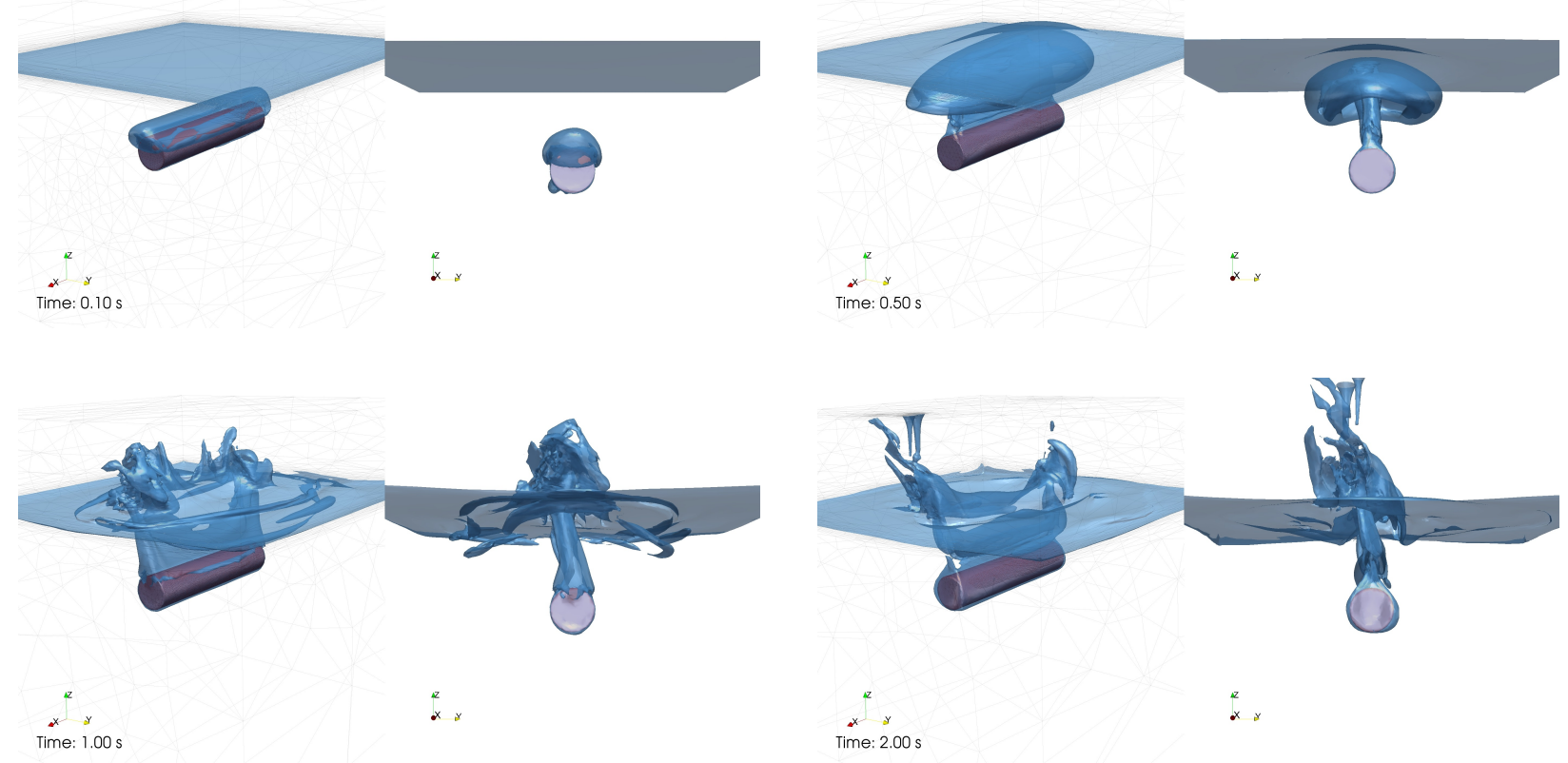

Figure 15: Cylinder at mid-height. Results at $\mathrm{t}=0.1,0.5,1$ and $2 \mathrm{~s}$. Perspective view (left), front view (right).
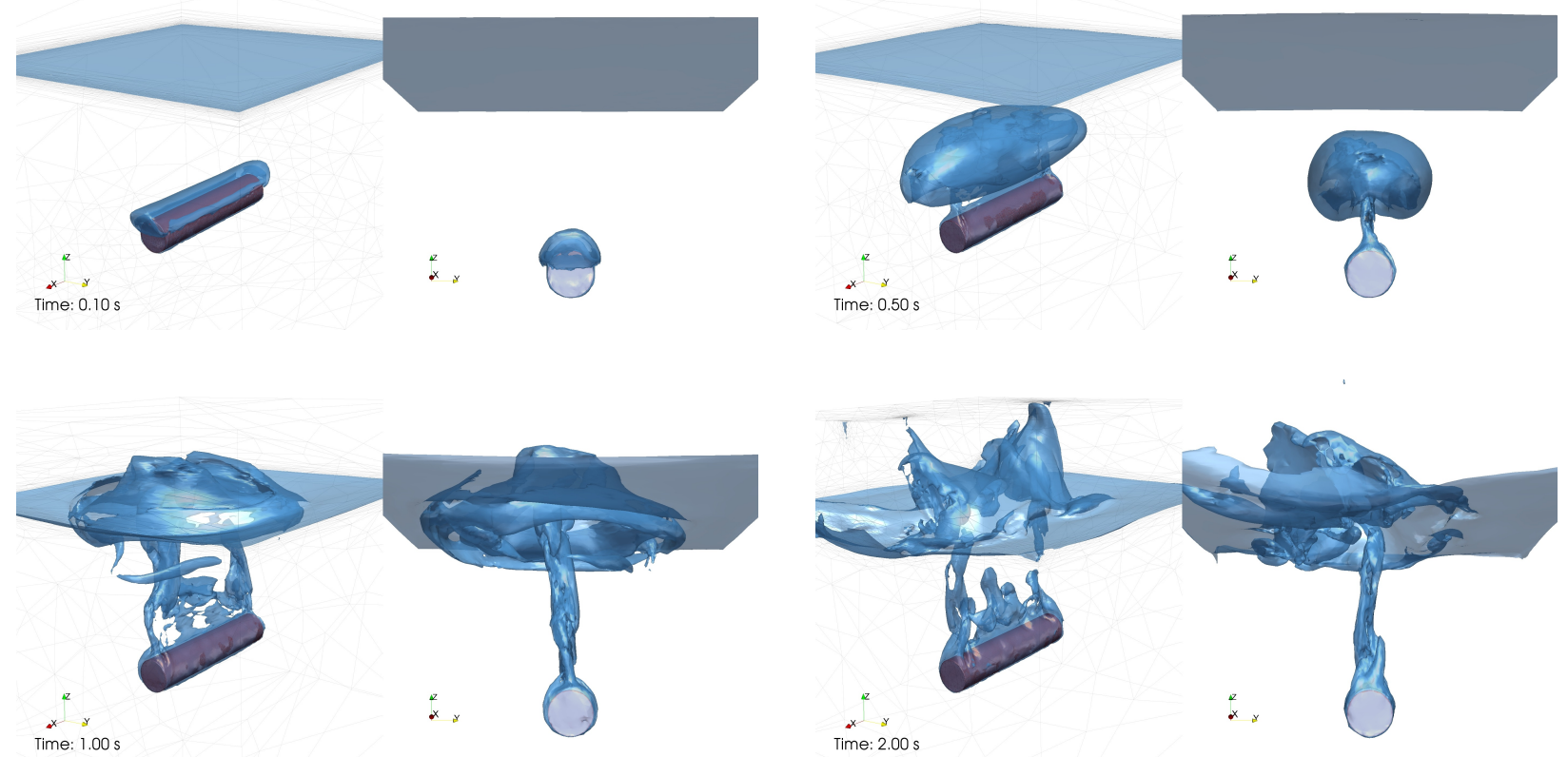

Figure 16: Cylinder at a quarter of the height. Results at $\mathrm{t}=0.1,0.5,1$ and $2 \mathrm{~s}$. Perspective view (left), front view (right).

Finally, Figure 19 shows this time that both the size of the hollow cylinder and its geometrical features seem to affect completely the flow. The boiling is well guided by the top surface with additional concentration 

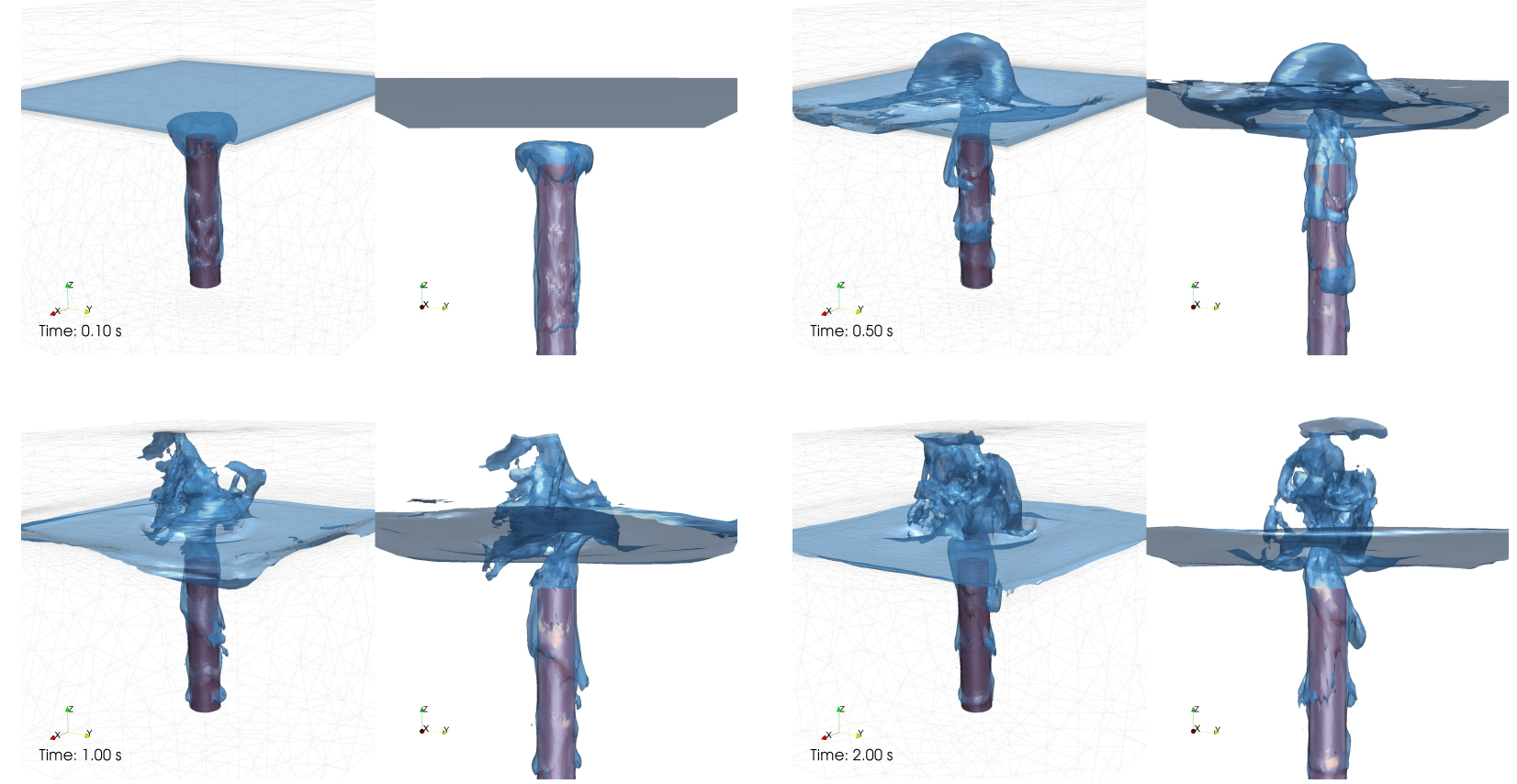

Figure 17: Vertical cylinder. Results at $\mathrm{t}=0.1,0.5,1$ and $2 \mathrm{~s}$. Perspective view (left), front view (right). several liquid-vapor phase changes during boiling as well as to handle easily optimal combination of quench parameters. 

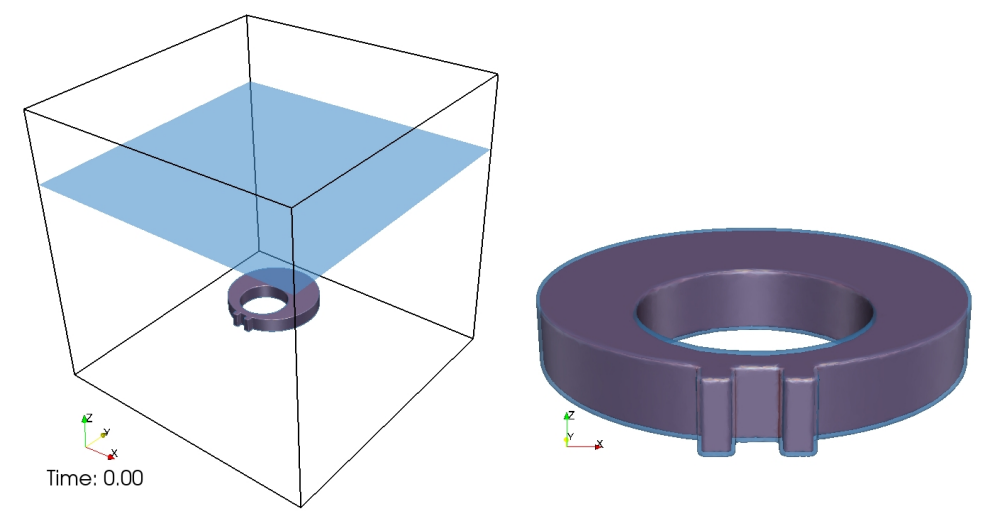

Figure 18: Hollow cylinder : Full domain with the ring (in red) and the gas-liquid interface in blue(left). Zoom on the ring (right)
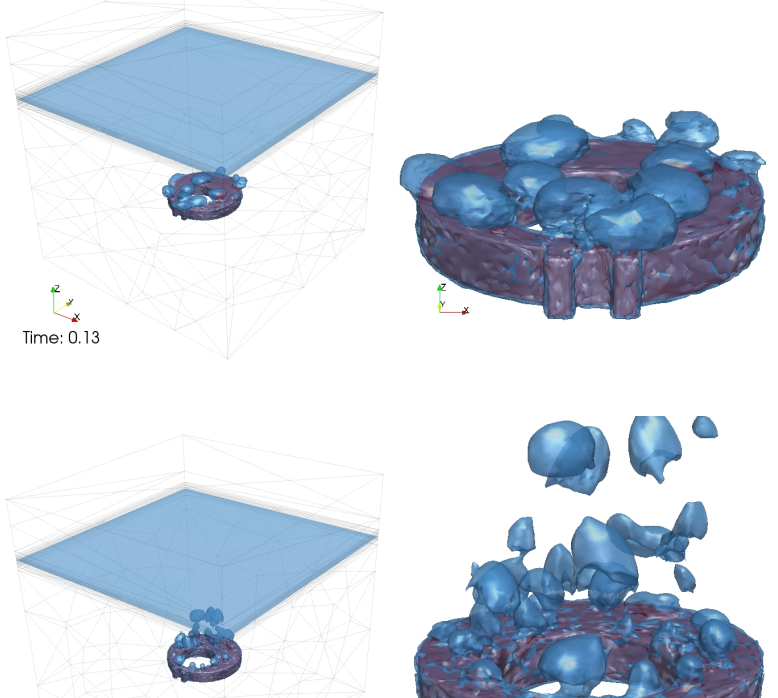

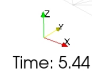

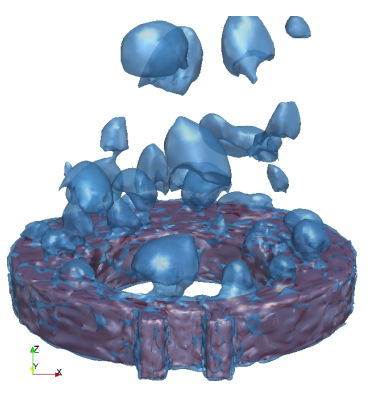

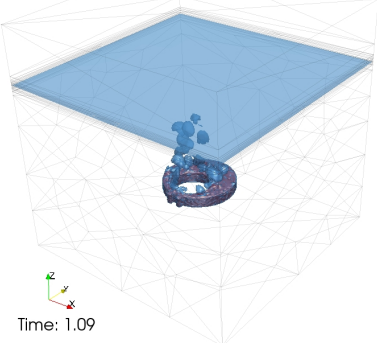
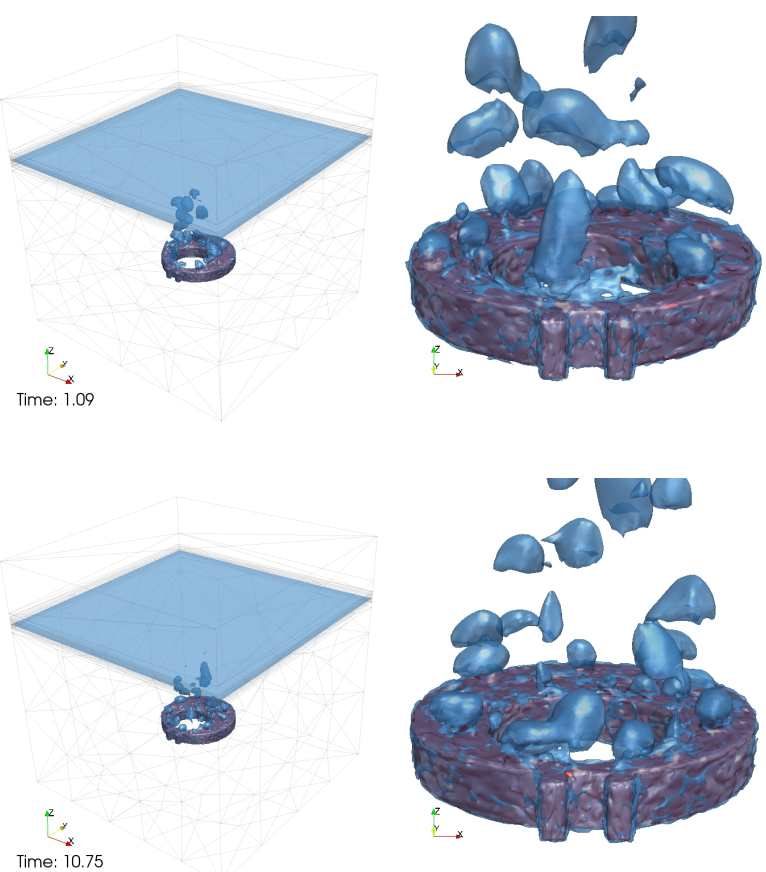

Figure 19: Hollow cylinder. Results at $\mathrm{t}=0.13,1.09,5.44$ and 10.75 s. Perspective view (left), front view (right). 


\subsection{Quenching of a solid, comparison with experimental data}

We consider a domain of size $0.60 \times 0.60 \times 0.40 \mathrm{~m}^{3}$, three-quarters full of water, in which a metallic sample of dimension $0.075 \times 0.075 \times 0.0015 \mathrm{~m}^{3}$ is immersed (see Figure 20). The temperature of the sample is $T_{\text {solid }}=880^{\circ} \mathrm{C}$ and the temperature of the water is $T_{\text {water }}=25^{\circ} \mathrm{C}$. A thermocouple is placed at the core of the metallic sample. From a practical point of view, due to the small thickness of the part, the temperature at the core reflects the behavior of the temperature field at the interface.

A free slip boundary condition is prescribed on all the walls. The motion of the vapor film is due to the buoyancy force and the surface tension force. The simulations are performed using the proposed adaptive meshing technique with 200,000 tetrahedra. The time step is set to $\Delta t=0.002 s$.

All the physical parameters related to each phase are presented in Table 3.

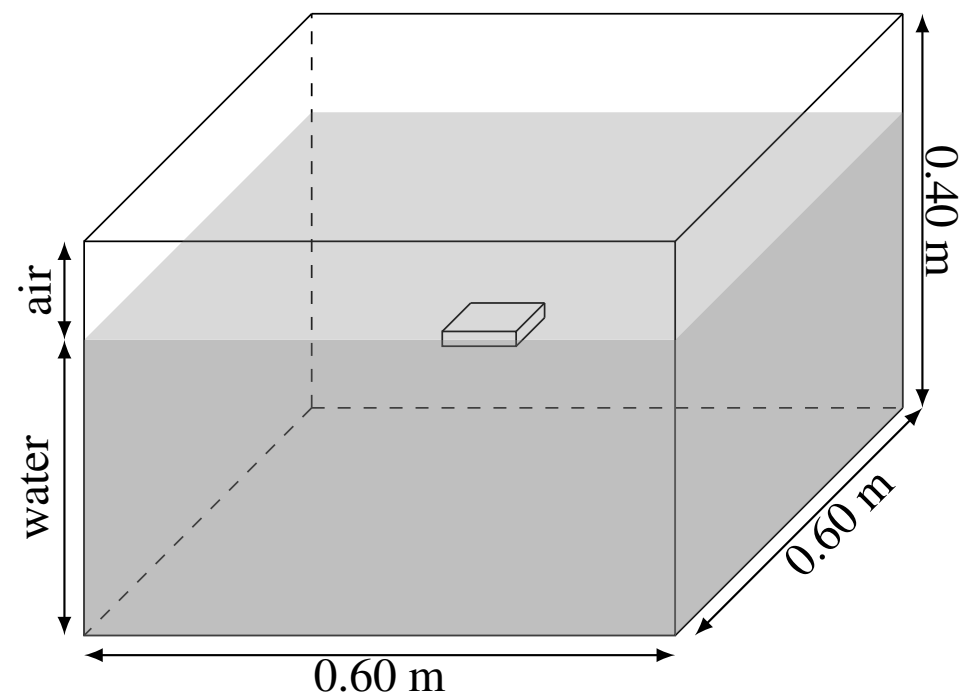

Figure 20: Set-up for the 3D industrial quenching

Table 3: Physical parameters defining the test case for the 3D industrial quenching

\begin{tabular}{cccccc} 
& $\rho\left[\mathrm{kg} / \mathrm{m}^{3}\right]$ & $\mu[\mathrm{Pa} \cdot \mathrm{s}]$ & $c_{p}[\mathrm{~J} /(\mathrm{kg} \cdot \mathrm{K})]$ & $\mathrm{k}[\mathrm{W} /(\mathrm{m} \cdot \mathrm{K})]$ & $\mathrm{L}_{\text {sat }}[\mathrm{J} / \mathrm{kg}]$ \\
\hline Vapor & 1.0 & $1.2 \times 10^{-5}$ & 2010 & 0.025 & $2.26 \times 10^{6}$ \\
Water & 1000 & $1.0 \times 10^{-3}$ & 4185 & 0.6 & \\
Solid & 8000 & & 435 & 11.4 & \\
\hline \hline
\end{tabular}




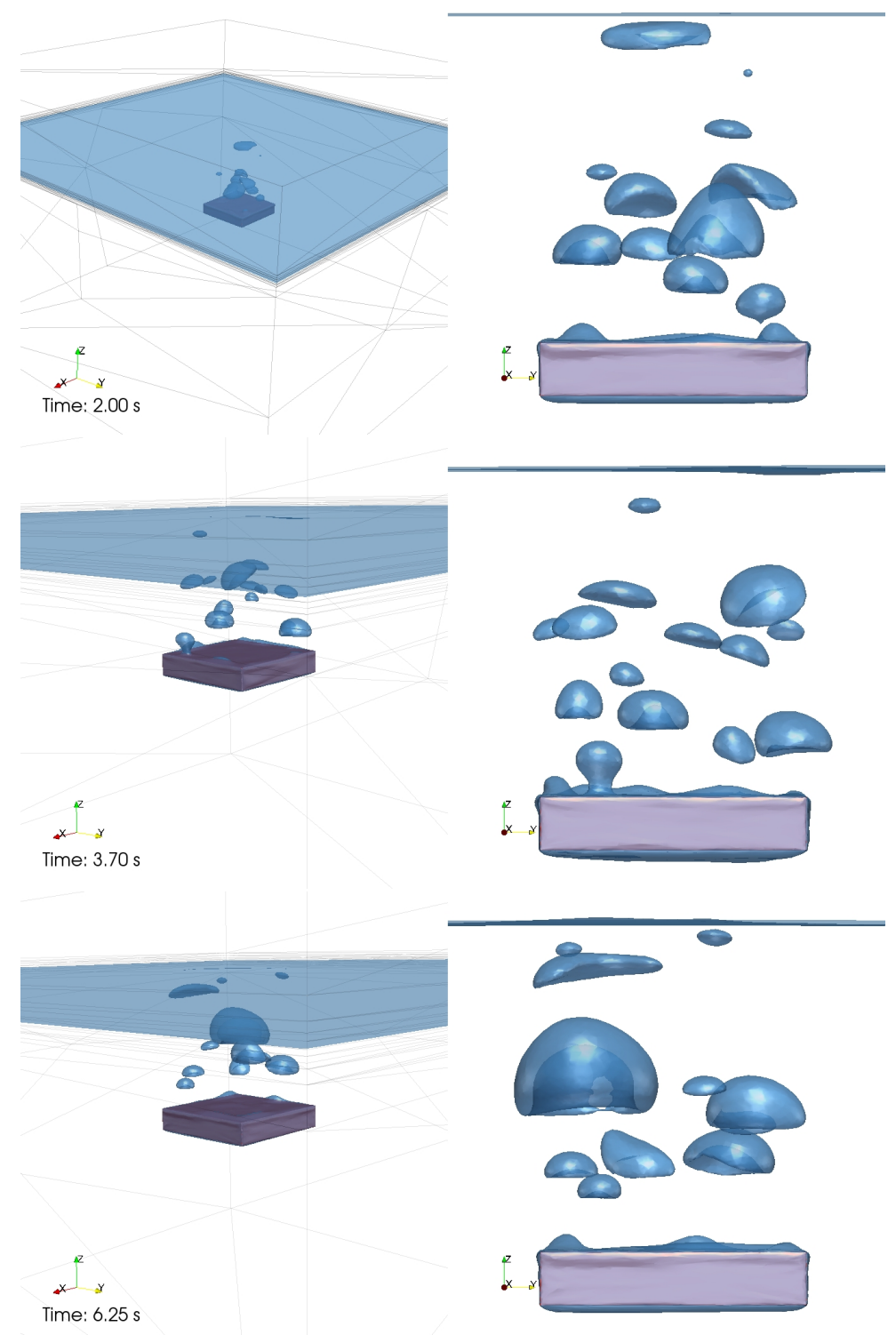

Figure 21: Industrial quenching. Results at $\mathrm{t}=2,3.70$ et $6.25 \mathrm{~s}$. Perspective view (left), front view (right). The zero isovalue of the level set is represented in blue.

Figure 22 represents a clip of the quenching tank. Water is depicted in blue and the solid in red. A slice enables to visualize the adaptive mesh over time. At the top left corner, the picture shows the initial mesh, mostly concentrated around the part and the free surface. As the simulation starts and bubbles form, the mesh is adapted automatically at the level set interface. Under the constraint of a fixed number of elements,

417 one can notice that the mesh is coarsened automatically at the bottom of the tank, where the variation of 418 the solutions is small.

419 Finally, Figure 23 shows a good agreement for the temperature evolution between the experimental data 

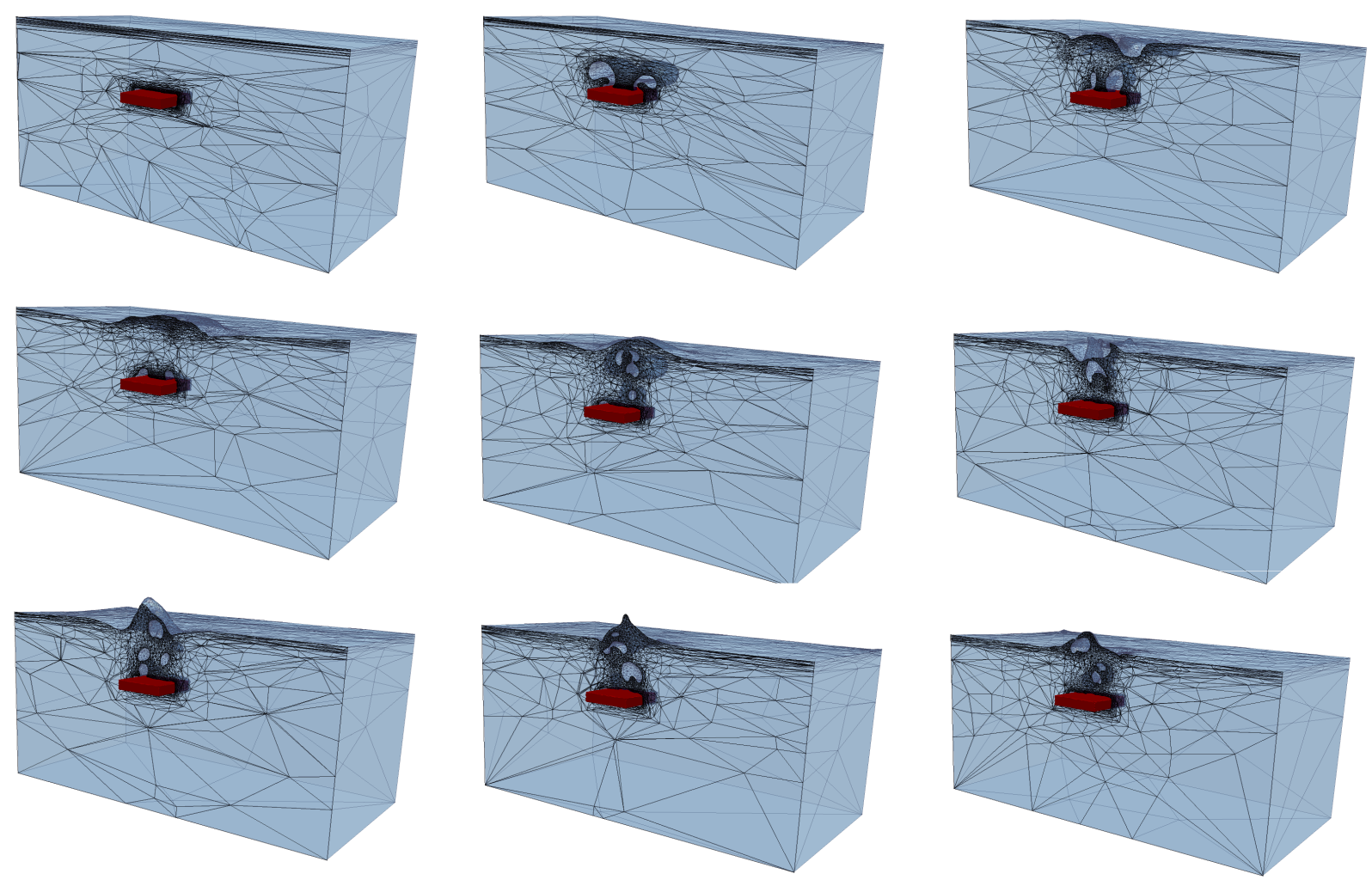

Figure 22: Industrial quenching: results at different instants. The solid is represented in red. A clip of the quenching tank, with visible adapted mesh.

420 and the numerical simulation. The strong coupling between the solid, the water and the vapor enables to 421 perform such simulation without the use of a heat transfer coefficient at the solid boundaries. The anisotropic 422 mesh adaptation enables to capture the large thermal gradient, the large jump at the interfaces as well as 423 the complexity of the flow. Simulations are performed using 100,000 to 400,000 elements. 


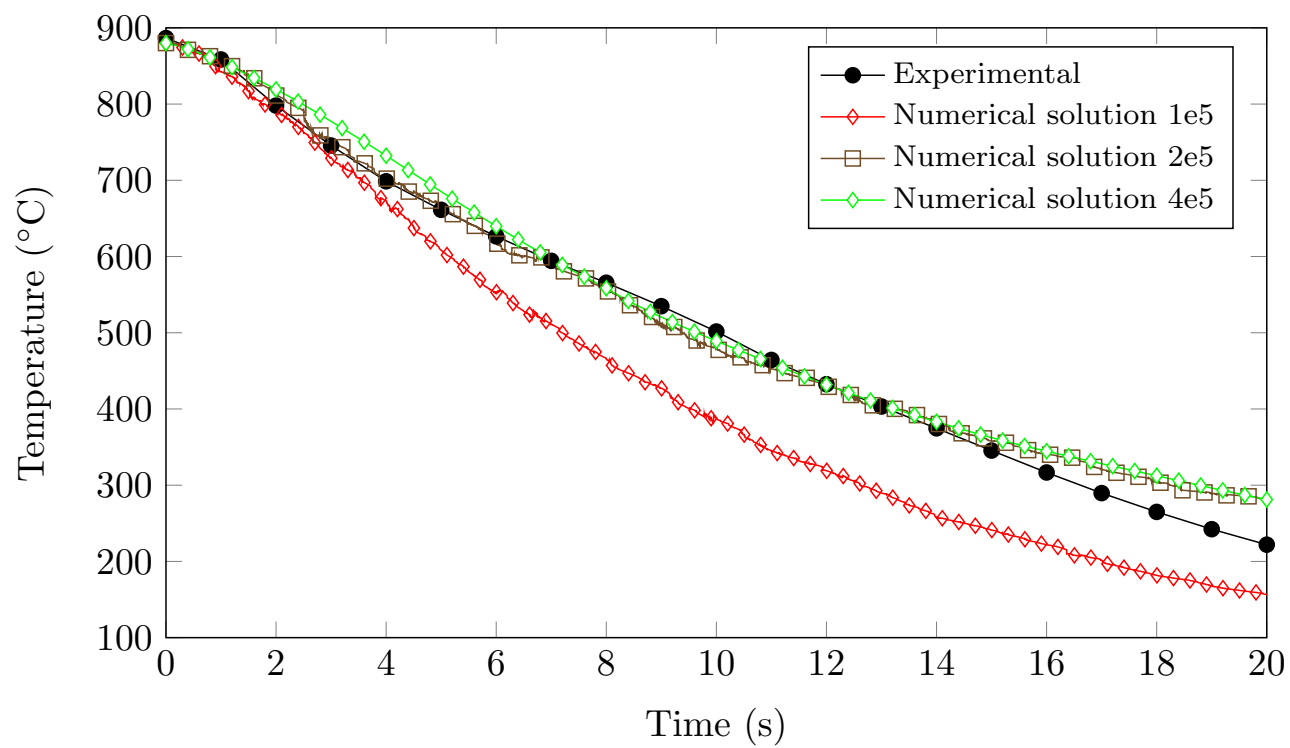

Figure 23: Evolution of the computed temperature at the core of the sample, sensitivity to the number of elements, comparison to experiments. 
We proposed an adaptive Eulerian framework for the numerical simulation of the phase change and the 426 evaporation that occurs at the interface between a heated solid surrounded by cooled liquid. We showed 427 that in order to model efficiently these phenomena, the gas-liquid phase changes, the vapor formation and 428 their dynamics, and the conjugate heat transfer must be solved simultaneously. Therefore, it replaces the 429 use of classical assumptions and ad-hoc transfer coefficients. We have demonstrated the efficiency of this 430 framework by performing challenging cases in 2D and 3D with comparisons including experimental data.

431 For the first time, the direct numerical simulation of the solid-liquid-gas interaction for industrial quenching 432 can be performed accurately.

\section{Acknowledgments}

434 The author gratefully acknowledges support from the companies involved in the ThosT project managed

435 by Science Computer and Consultants (SCC): Areva NP, Aubert \& Duval, Faurecia, Industeel CRMC 436 (Arcelor Mittal), Lisi Aerospace and Snecma/Safran. 


\section{References}

[1] A. Esmaeeli, G. Tryggvason, Computations of film boiling. Part I: numerical method , International Journal of Heat and Mass Transfer 47 (25) (2004) $5451-5461$.

[2] A. Esmaeeli, G. Tryggvason, Computations of film boiling. Part II: multi-mode film boiling , International Journal of Heat and Mass Transfer 47 (25) (2004) 5463 - 5476.

[3] D. Juric, G. Tryggvason, Computations of boiling flows, International Journal of Multiphase Flow 24 (3) (1998) 387 - 410.

[4] G. Tryggvason, A. Esmaeeli, N. Al-Rawahi, Direct numerical simulations of flows with phase change, Computers \& Structures 83 (67) (2005) $445-453$.

[5] M. Irfan, M. Muradoglu, A front tracking method for direct numerical simulation of evaporation process in a multiphase system, Journal of Computational Physics 337 (2017) 132 - 153.

[6] F. Gibou, R. Fedkiw, S. Osher, A review of level-set methods and some recent applications, Journal of Computational Physics 353 (2018) $82-109$.

[7] J. Papac, A. Helgadottir, C. Ratsch, F. Gibou, A level set approach for diffusion and Stefan-type problems with Robin boundary conditions on quadtree/octree adaptive Cartesian grids, Journal of Computational Physics 233 (2013) 241 261.

[8] S. W. Welch, J. Wilson, A Volume of Fluid Based Method for Fluid Flows with Phase Change, Journal of Computational Physics 160 (2) (2000) $662-682$.

[9] S. W. Welch, Local simulation of two-phase flows including interface tracking with mass transfer, Journal of Computational Physics 121 (1) (1995) $142-154$.

[10] Y. Sato, B. Nieno, A sharp-interface phase change model for a mass-conservative interface tracking method, Journal of Computational Physics 249 (2013) 127 - 161.

[11] S. Hardt, F. Wondra, Evaporation model for interfacial flows based on a continuum-field representation of the source terms, Journal of Computational Physics 227 (11) (2008) 5871 - 5895.

[12] S. Tanguy, T. Ménard, A. Berlemont, A level set method for vaporizing two-phase flows, Journal of Computational Physics 221 (2) (2007) $837-853$.

[13] J. Schlottke, B. Weigand, Direct numerical simulation of evaporating droplets, Journal of Computational Physics 227 (10) (2008) $5215-5237$.

[14] J. Lee, G. Son, H. Y. Yoon, Numerical simulation of the quenching process in liquid jet impingement, International Communications in Heat and Mass Transfer 61 (2015) 146 - 152.

[15] M. Yazdani, T. Radcliff, M. Soteriou, A. A. Alahyari, A high-fidelity approach towards simulation of pool boiling, Physics of Fluids 28 (1) (2016) 012111.

[16] V. Mihalef, B. Unlusu, D. Metaxas, M. Sussman, M. Y. Hussaini, Physics based boiling simulation, in: Proceedings of the 2006 ACM SIGGRAPH/Eurographics Symposium on Computer Animation, SCA '06, Eurographics Association, Aire-la-Ville, Switzerland, Switzerland, 2006, pp. 317-324.

[17] L. Anumolu, M. F. Trujillo, Gradient augmented level set method for phase change simulations, Journal of Computational Physics 353 (2018) $377-406$.

[18] H. Chen, C. Min, F. Gibou, A numerical scheme for the Stefan problem on adaptive Cartesian grids with supralinear convergence rate, Journal of Computational Physics 228 (16) (2009) $5803-5818$.

[19] C. R. Kharangate, I. Mudawar, Review of computational studies on boiling and condensation, International Journal of Heat and Mass Transfer 108, Part A (2017) 1164 - 1196.

[20] J. Liu, C. M. Landis, H. Gomez, T. J. Hughes, Liquid-vapor phase transition: Thermomechanical theory, entropy stable numerical formulation, and boiling simulations, Computer Methods in Applied Mechanics and Engineering 297 (2015) 476 $-553$. 
[21] J. Bueno, H. Gomez, Liquid-vapor transformations with surfactants. Phase-field model and Isogeometric Analysis , Journal of Computational Physics 321 (2016) $797-818$.

[22] J. Bueno, C. Bona-Casas, Y. Bazilevs, H. Gomez, Interaction of complex fluids and solids: theory, algorithms and application to phase-change-driven implosion, Computational Mechanics 55 (6) (2015) 1105-1118.

[23] R. Saurel, R. Abgrall, A Multiphase Godunov Method for Compressible Multifluid and Multiphase Flows, Journal of Computational Physics 150 (2) (1999) 425 - 467.

[24] R. Saurel, F. Petitpas, R. Abgrall, Modelling phase transition in metastable liquids: application to cavitating and flashing flows, Journal of Fluid Mechanics 607 (2008) 313350.

[25] M. Khalloufi, Y. Mesri, R. Valette, E. Massoni, E. Hachem, High fidelity anisotropic adaptive variational multiscale method for multiphase flows with surface tension, Computer Methods in Applied Mechanics and Engineering 307 (2016) $44-67$.

[26] L. Marioni, M. Khalloufi, F. Bay, E. Hachem, Two-fluid flow under the constraint of external magnetic field: revisiting the dam-break benchmark, International Journal of Numerical Methods for Heat and Fluid Flow 29 (2016) 1-17.

[27] R. Valette, E. Hachem, M. Khalloufi, A. Pereira, M. Mackley, S. Butler, The effect of viscosity, yield stress, and surface tension on the deformation and breakup profiles of fluid filaments stretched at very high velocities, Journal of NonNewtonian Fluid Mechanics 263 (2019) 130 - 139.

[28] C. Bahbah, M. Khalloufi, A. Larcher, Y. Mesri, T. Coupez, R. Valette, E. Hachem, Conservative and adaptive level-set method for the simulation of two-fluid flows, Computers \& Fluids 191 (2019) 104223.

[29] E. Hachem, M. Khalloufi, J. Bruchon, R. Valette, Y. Mesri, Unified adaptive Variational MultiScale method for two phase compressible-incompressible flows, Computer Methods in Applied Mechanics and Engineering 308 (2016) 238 - 255.

[30] S. Riber, R. Valette, Y. Mesri, E. Hachem, Adaptive variational multiscale method for bingham flows, Computers \& Fluids 138 (2016) $51-60$.

[31] Y. Mesri, M. Khalloufi, E. Hachem, On optimal simplicial 3D meshes for minimizing the Hessian-based errors , Applied Numerical Mathematics 109 (2016) 235 - 249.

[32] T. Coupez, Metric construction by length distribution tensor and edge based error for anisotropic adaptive meshing, Journal of Computational Physics 230 (7) (2011) 2391-2405.

[33] J. Sari, F. Cremonesi, M. Khallouf, F. Cauneau, P. Meliga, Y. Mesri, E. Hachem, Anisotropic adaptive stabilized finite element solver for RANS models, International Journal for Numerical Methods in Fluids 86 (11) (2018) $717-736$.

[34] S. Osher, R. Fedkiw, Level set methods and dynamic implicit surfaces, Springer, 2003.

[35] M. Sussman, P. Smereka, S. Osher, A level set approach for computing solutions to incompressible two-phase flow, Journal of Computational Physics 114 (1) (1994) 146 - 159.

[36] R. Denis, Modelisation and simulation of Leidenfrost effect in micro-droplets, Phd thesis, Université de Grenoble (Nov. 2012).

[37] M. S. Lee, A. Riaz, V. Aute, Direct numerical simulation of incompressible multiphase flow with phase change, Journal of Computational Physics 344 (2017) $381-418$.

[38] J. Brackbill, D. Kothe, C. Zemach, A continuum method for modeling surface tension, Journal of Computational Physics 100 (2) (1992) $335-354$.

[39] S. Hysing, A new implicit surface tension implementation for interfacial flows, International Journal for Numerical Methods in Fluids 51 (6) (2006) 659-672.

[40] G. C. Buscaglia, R. F. Ausas, Variational formulations for surface tension, capillarity and wetting, Computer Methods in Applied Mechanics and Engineering 200 (45 - 46) (2011) 3011 - 3025.

[41] V. Karyofylli, M. Frings, S. Elgeti, M. Behr, Simplex space-time meshes in two-phase flow simulations, International Journal for Numerical Methods in Fluids 86 (3) (2018) 218-230. 
[42] T. Hughes, Multiscale phenomena: Green's functions, the Dirichlet-to-Neumann formulation, subgrid scale models, bubbles and the origins of stabilized methods, Computer Methods in Applied Mechanics and Engineering 127 (1 - 4) (1995) 387 -401 .

[43] T. Hughes, G. R. Feijo, L. Mazzei, J.-B. Quincy, The variational multiscale method-a paradigm for computational mechanics, Computer Methods in Applied Mechanics and Engineering 166 (1 - 2) (1998) 3 - 24.

[44] K. Nakshatrala, D. Turner, K. Hjelmstad, A. Masud, A stabilized mixed finite element method for darcy flow based on a multiscale decomposition of the solution, Computer Methods in Applied Mechanics and Engineering 195 (33 - 36) (2006) $4036-4049$.

[45] G. Scovazzi, Lagrangian shock hydrodynamics on tetrahedral meshes: A stable and accurate variational multiscale approach, Journal of Computational Physics 231 (24) (2012) 8029 - 8069.

[46] U. Rasthofer, V. Gravemeier, Multifractal subgrid-scale modeling within a variational multiscale method for large-eddy simulation of turbulent flow, Journal of Computational Physics 234 (2013) $79-107$.

[47] E. Castillo, R. Codina, Variational multi-scale stabilized formulations for the stationary three-field incompressible viscoelastic flow problem, Computer Methods in Applied Mechanics and Engineering 279 (2014) 579 - 605.

[48] E. Hachem, B. Rivaux, T. Kloczko, H. Digonnet, T. Coupez, Stabilized finite element method for incompressible flows with high reynolds number, Journal of Computational Physics 229 (23) (2010) 8643-8665.

[49] L. Formaggia, S. Perotto, Anisotropic error estimates for elliptic problems, Numerische Mathematik 94 (1) (2003) 67-92.

[50] J. Hoffman, C. Johnson, Adaptive finite element methods for incompressible fluid flow, Error Estimation and Adaptive Discretization in Computational Fluid Dynamics (Ed. T. J. Barth and H. Deconinck), Lecture Notes in Computational Science and Engineering Vol.25, Springer-Verlag Publishing, Heidelberg, 2003.

[51] G. Legrain, N. Moës, Adaptive anisotropic integration scheme for high-order fictitious domain methods: Application to thin structures, International Journal for Numerical Methods in Engineering 114 (8) (2018) 882-904.

[52] G. Kunert, R. Verfürth, Edge residuals dominate a posteriori error estimates for linear finite element methods on anisotropic triangular and tetrahedral meshes, Numer. Math. 86 (2) (2000) 283-303.

[53] F. Gibou, L. Chen, D. Nguyen, S. Banerjee, A level set based sharp interface method for the multiphase incompressible Navier-Stokes equations with phase change, Journal of Computational Physics 222 (2) (2007) 536 - 555.

[54] M. W. Akhtar, S. J. Kleis, Boiling flow simulations on adaptive octree grids, International Journal of Multiphase Flow 53 (2013) $88-99$.

[55] P. J. Berenson, Film-Boiling Heat Transfer From a Horizontal Surface, Journal of Heat Transfer 83 (3) (1961) 351 - 356.

[56] V. Klimenko, Film boiling on a horizontal plate - new correlation, International Journal of Heat and Mass Transfer 24 (1) (1981) $69-79$. 